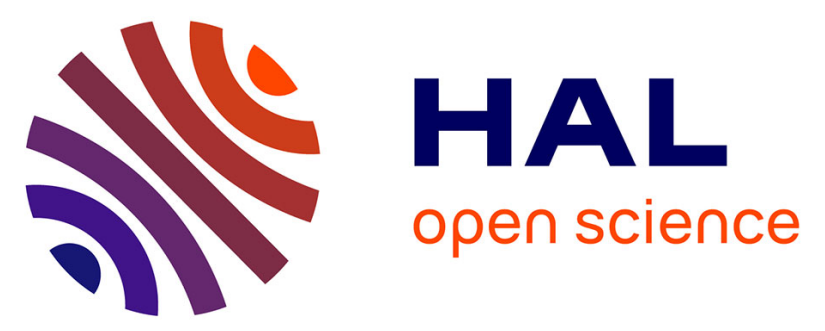

\title{
Centralized versus Decentralized Multi-Cell Resource and Power Allocation for Multiuser OFDMA Networks
}

Mohamad Yassin, Samer Lahoud, Kinda Khawam, Marc Ibrahim, Dany Mezher, Bernard Cousin

\section{- To cite this version:}

Mohamad Yassin, Samer Lahoud, Kinda Khawam, Marc Ibrahim, Dany Mezher, et al.. Centralized versus Decentralized Multi-Cell Resource and Power Allocation for Multiuser OFDMA Networks. Computer Communications, 2017, 107, pp.112 - 124. 10.1016/j.comcom.2017.04.002 . hal-01539926

\section{HAL Id: hal-01539926 \\ https://hal.science/hal-01539926}

Submitted on 15 Jun 2017

HAL is a multi-disciplinary open access archive for the deposit and dissemination of scientific research documents, whether they are published or not. The documents may come from teaching and research institutions in France or abroad, or from public or private research centers.
L'archive ouverte pluridisciplinaire HAL, est destinée au dépôt et à la diffusion de documents scientifiques de niveau recherche, publiés ou non, émanant des établissements d'enseignement et de recherche français ou étrangers, des laboratoires publics ou privés. 


\title{
Centralized versus Decentralized Multi-Cell Resource and Power Allocation for Multiuser OFDMA Networks
}

\author{
Mohamad Yassina,b,*, Samer Lahoud ${ }^{\mathrm{a}}$, Kinda Khawam ${ }^{\mathrm{c}}$, Marc Ibrahim $^{\mathrm{b}}$, \\ Dany Mezher ${ }^{\mathrm{b}}$, Bernard Cousin ${ }^{\mathrm{a}}$ \\ ${ }^{a}$ University of Rennes 1, IRISA, Campus de Beaulieu, 35042 Rennes, France \\ ${ }^{b}$ Saint Joseph University of Beirut, ESIB, CST, Mar Roukoz, Lebanon \\ ${ }^{c}$ University of Versailles, 45 Av. des Etats-Unis, 78035 Versailles, France
}

\begin{abstract}
The exponential growth in the usage of mobile networks along with the increasing number of User Equipments (UEs) are exacerbating the scarcity of frequency resources. Dense frequency reuse on the downlink of multiuser Orthogonal Frequency Division Multiple Access networks leads to severe Inter-Cell Interference (ICI) problems. Resource and power allocation techniques are required to alleviate the harmful impact of ICI. Contrarily to the existing techniques that consider single-cell resource and power allocation problem without taking ICI into account, we formulate a centralized downlink multi-cell joint resource and power allocation problem. The objective is to maximize system throughput while guaranteeing throughput fairness between UEs. We demonstrate that the joint problem is separable into two independent problems: a resource allocation problem and a power allocation problem. Lagrange duality theory is used to solve the centralized power allocation problem. We also tackle the resource and power allocation problem differently by addressing it in a decentralized manner. We propose a non-cooperative downlink power allocation approach based on game theory. The players are the base stations, and each base station seeks to maximize its own utility function. We investigate the convergence of our proposed centralized and decentralized approaches, and we
\end{abstract}

\footnotetext{
*Corresponding author

Email address: mohamad.yassin@usj.edu.lb (Mohamad Yassin)
} 
compare their performance with that of state-of-the-art approaches.

Keywords: Convex optimization, resource and power allocation, inter-cell interference, ICIC, OFDMA

\section{Introduction}

Multiuser Orthogonal Frequency Division Multiple Access (OFDMA) networks, such as the Third Generation Partnership Project (3GPP) Long Term Evolution (LTE) [1] and LTE-Advanced (LTE-A) [2] networks, are able to avoid the negative impact of multipath fading and intra-cell interference, by virtue of the orthogonality between subcarrier frequencies. Nevertheless, Inter-Cell Interference (ICI) problems arise on the downlink of dense frequency reuse networks due to simultaneous transmissions on the same frequency resources. System performance is interference-limited, since the achievable throughput is reduced due to ICI.

Fractional Frequency Reuse (FFR) [3] and Soft Frequency Reuse (SFR) [4] were introduced to avoid the harmful impact of ICI on system performance, by applying static rules on Resource Block (RB) usage and power allocation between cell-center and cell-edge users. Heuristic Inter-Cell Interference Coordination (ICIC) techniques are proposed to achieve ICI mitigation without severe degradation of the overall system throughput. For instance, authors of $[5,6]$ propose suboptimal solution for the resource allocation problem. The objective is to minimize ICI by exploiting User Equipment (UE) diversity to maximize system throughput. They propose a two-level algorithm that operates at the evolved-NodeBs (eNodeBs) and at a central controller connected to several eNodeBs. In [7], a heuristic power allocation algorithm is introduced to reduce energy consumption and to improve cell-edge UEs throughput. It has been proven that the proposed algorithm reduces power consumption without reducing the achievable throughput. Moreover, it mitigates ICI and increases the achievable throughput for cell-edge UEs.

Beside heuristic resource and power allocation algorithms [8], convex opti- 
mization is used to improve the performance of multiuser OFDMA networks, and to alleviate the negative impact of ICI on UE throughput. Resource and power allocation problem is usually formulated as nonlinear optimization problem, where the objective consists in maximizing system throughput, spectral efficiency, or energy efficiency, with constraints on the minimum throughput per UE or other Quality of Service (QoS) parameters [9]. The exponential growth in the usage of mobile networks along with the increasing number of UEs are exacerbating the scarcity of frequency resources.

The majority of state-of-the-art contributions formulate the resource and power allocation problem for a single cell network [10,11, 12], or do not consider the impact of ICI on system performance. For instance, the tradeoff between spectral efficiency and energy efficiency is addressed in [12], and a low-complexity suboptimal algorithm is proposed to allocate RBs for practical 40 applications of the tradeoff. However, the system model consists of a single cell OFDMA network, where one subcarrier is assigned to at most one UE. Therefore, ICI problems are not considered. In this article, we formulate the joint resource and power allocation problem for the downlink of multiuser OFDMA networks, as a centralized multi-cell optimization problem. Inter-cell interference is taken into account, and throughput fairness between the different users is guaranteed. We prove that our joint problem is separable into two independent optimization problems: a resource allocation problem and a power allocation problem. Our objective is to maximize system throughput, while satisfying constraints related to resource usage, Signal-to-Interference and Noise Ratio ${ }_{50}$ (SINR), and power allocation. We also propose a decentralized power allocation approach that does not rely on centralized controllers. Each base station maximizes its own utility function in a distributed manner. We evaluate the performance of the proposed approaches, and we compare their performance with state-of-the-art resource and power allocation approaches.

55 The remainder of this article is organized as follows. In section II, we describe the limitations of the existing state-of-the-art approaches. In section III, system model is presented followed by our joint resource and power allocation 
problem formulation. The joint problem is decomposed into two independent problems in section IV: a resource allocation problem and a power allocation problem. We also demonstrate the convexity of the formulated problems. In section $\mathrm{V}$, we solve both resource and power allocation problems using the Lagrange duality theory. Our decentralized power allocation approach is introduced in section VI. Then, we investigate the convergence of the centralized and the decentralized approaches in section VII, where we also provide comparisons with other approaches. Section VIII concludes this article and summarizes our main contributions.

\section{Related Work}

\subsection{State-of-the-Art Contributions}

For a given multiuser OFDMA network, resource and power allocation problem is formulated as a centralized optimization problem [10,11, 12]. Centralized inter-cell coordination is therefore required to find the optimal solution, where the necessary information about SINR, power allocation, and resource usage are sent to a centralized coordination entity.

In [13], the multi-cell optimization problem is decomposed into two distributed optimization problems. The objective of the first problem is to minimize the transmission power allocated for cell-edge UEs, while guaranteeing a minimum throughput for each UE. RB and power are allocated to cell-edge UEs so that they satisfy their minimum required throughput. The remaining RBs and the remaining transmission power are uniformly allocated to cell-center tion strategy that maximizes the achievable throughput for cell-center UEs. An improved version of this adaptive ICIC technique is proposed in [14], where resource allocation for cell-edge UEs is performed depending on their individual channel conditions. However, the main disadvantage of this adaptive ICIC technique and the proposed improvement is that they do not consider the impact of ICI between adjacent cells when power allocation is performed. Each cell solves 
its own optimization problem without requesting additional information from its neighboring cells.

Resource and power allocation for a cluster of coordinated OFDMA cells 90 are studied in [15]. Energy efficiency is maximized under constraints related to the downlink transmission power. However, noise-limited regime is considered, and ICI is neglected. Moreover, energy-efficient resource allocation for OFDMA systems is investigated in [16], where generalized and individual energy efficiencies are defined for the downlink and the uplink of the OFDMA system, respectively. Properties of the energy efficiency objective function are studied, then a low-complexity suboptimal algorithm is introduced to reduce the computational burden of the optimal solution. Subcarrier assignment is made easier using heuristic algorithms. Authors of [17] consider the joint resource allocation, power allocation, and Modulation and Coding Scheme (MCS) selection problem. The joint optimization problem is separated into resource allocation and power allocation problems, and suboptimal algorithms are proposed. Another low complexity suboptimal resource allocation algorithm is proposed in [18]. The objective consists in maximizing the achievable throughput, under constraints related to resource usage in the different cells. Cooperation between adjacent cells is needed. A multi-cell resource allocation approach for OFDMA systems with decode-and-forward relaying is proposed in [19], where an interference constraint is introduced along with time sharing variables. Although this approach guarantees throughput fairness between the different users, the spectral efficiency is reduced since the cells are not allowed to use the available spectrum during $100 \%$ of the time due to time sharing between base stations and relays.

Minimizing energy consumption and maximizing spectral efficiency in multiuser OFDMA networks cannot be achieved simultaneously. Energy-bandwidth tradeoff is studied in [20], where authors consider the total energy consumption versus the end-to-end rate in wireless multihop networks. For an arbitrary placement of wireless nodes, resource and power allocation that minimizes the energy level required to achieve a given data rate is found. However, interference-free 
resource allocation is considered, and the impact of ICI on system performance is not taken into account.

\subsection{Our Contributions}

The majority of state-of-the-art contributions that formulate spectral efficiency or energy efficiency problems as centralized optimization problems, neglect the impact of ICI on system performance $[10,11,12]$, or introduce suboptimal approaches to solve resource and power allocation problems [21, 22, 23]. Moreover, performance comparisons are not made with other distributed heuristic ICIC algorithms, that are usually characterized by a lower computational complexity. In our work, we consider the multi-cell downlink resource and power allocation problem, where the objective is to maximize system throughput while guaranteeing throughput fairness between the different UEs. Moreover, ICI is taken into account when solving the centralized resource and power allocation problem. We also formulate a decentralized non-cooperative power allocation approach based on game theory. The players are the cells, and each cell seeks maximizing its own utility function independently of the other cells in the network. We investigate the convergence of both centralized and decentralized approaches, and we compare their performance with that of the frequency reuse-1 model, the frequency reuse-3 model, FFR, and SFR techniques. Our major contributions are summarized as follows:

- Propose an original formulation of the centralized joint resource and power allocation problem: instead of considering a single cell OFDMA network, we formulate our problem for a multi-cell OFDMA network, taking ICI problems into account. The objective is to maximize the mean rate per UE, and ensure a proportional fair rate for all the active UEs.

- Decompose the joint downlink resource and power allocation problem into two independent problems, and solve the centralized power allocation problem using Lagrange duality theory and subgradient projection method. 
- Formulate a novel decentralized super-modular game for resource and power allocation, and propose a best response algorithm to attain the Nash Equilibrium. Then, solve the decentralized power allocation problem using subgradient projection method.

- Validate the convergence of the proposed centralized and decentralized approaches and evaluate their performance in comparison with broadly adopted state-of-the-art approaches.

\section{System Model and Problem Formulation}

155

\subsection{System Model}

We consider the downlink of a multiuser OFDMA system that consists of $I$ adjacent cells and $K$ active UEs. Let $\mathcal{I}=\{1,2, \ldots, I\}$ denote the the set of cells, and $\mathcal{K}=\{1,2, \ldots, K\}$ the total set of active UEs. We also define $K(i)$ as the number of UEs served by cell $i$. Thus, we have $\sum_{i=1}^{I} K(i)=K$. The set of available RBs in each cell is denoted by $\mathcal{N}=\{1,2, \ldots, N\}$.

In OFDMA networks, system spectrum is divided into several channels, where each channel consists of a number of consecutive orthogonal OFDM subcarriers [24]. An RB is the smallest scheduling unit. It consists of 12 consecutive subcarriers in the frequency domain, and seven OFDM symbols with normal cyclic prefix in the time domain [25] (or six OFDM symbols with extended cyclic prefix). Frequency resources are allocated to UEs each Transmit Time Interval (TTI), which is equal to $1 \mathrm{~ms}$. When the frequency reuse- 1 model is applied along with homogeneous power allocation, each RB is allocated the same downlink transmission power $\frac{P_{\max }}{N}$, where $P_{\max }$ denotes the maximum downlink transmission power per cell. The signal to interference and noise ratio for a UE $k$ attached to cell $i$ and allocated $\operatorname{RB} n$ is given by:

$$
\sigma_{k, i, n}=\frac{\pi_{i, n} G_{k, i, n}}{N_{0}+\sum_{i^{\prime} \neq i} \pi_{i^{\prime}, n} G_{k, i^{\prime}, n}}
$$


where $\pi_{i, n}$ is the downlink transmission power allocated by cell $i$ to $\mathrm{RB} n, G_{k, i, n}$ denotes channel gain for UE $k$ attached to cell $i$ and allocated RB $n$, and $N_{0}$ is the thermal noise power. Indexes $i$ and $i^{\prime}$ refer to useful and interfering signals respectively. In our work, we rely on perfect channel state information to infer the SINR. Authors of [26] provide models to account for imperfect channel state and study the impact on energy efficiency. Notations, symbols, parameters, and variables used within this document are reported in Table 1.

Table 1: Sets, parameters and variables in the article

\begin{tabular}{ll}
\hline$i$ & Index of cell \\
$k$ & Index of UE \\
$n$ & Index of RB \\
$\mathcal{I}$ & Set of cells \\
$\mathcal{K}$ & Total set of UEs \\
$\mathcal{K}(i)$ & Set of UEs associated to cell $i$ \\
$\mathcal{N}$ & Set of RBs \\
$\rho_{k, i, n}$ & Peak rate of UE $k$ associated with RB $n$ on cell $i$ \\
$\pi_{i, n}$ & Transmit power of cell $i$ on RB $n$ \\
$G_{k, i, n}$ & Channel gain for UE $k$ over RB $n$ on cell $i$ \\
$N_{0}$ & Thermal noise density \\
$\theta_{k, n}$ & Percentage of time UE $k$ is associated with RB $n$ \\
$\eta$ & Total system achievable mean rate \\
$\sigma_{k, i, n}$ & SINR for UE $k$ over RB $n$ on cell $i$ \\
$P_{\max }$ & Maximum DL transmission power per cell \\
$\pi_{\min }$ & Minimum DL transmission power per RB \\
$\mathcal{I}^{\prime}(i)$ & Set of neighboring cells for cell $i$ \\
\hline
\end{tabular}

\subsection{Problem Formulation}

\subsubsection{Centralized Multi-Cell Optimization Problem}

We define $\theta_{k, n}$ as the percentage of time during which UE $k$ is associated with RB $n . \theta_{k, n}, \forall k \in \mathcal{K}, \forall n \in \mathcal{N}$, and $\pi_{i, n}, \forall i \in \mathcal{I}, \forall n \in \mathcal{N}$, are the optimization variables of the joint resource and power allocation problem. Our objective is to manage resource and power allocation in a manner that maximizes system throughput and guarantees throughput fairness between the different UEs. The standard approach is to have integer scheduling variables, while in our problem formulation, $\theta_{k, n}$ and $\pi_{i, n}$ are continuous variables. In fact, using continuous variables will decrease the computation time and the complexity of the problem 
without losing generality. A simple way of implementing the solution is to extend the Round-Robin scheduler in a way to allocate equal time shares to the users in the cell on each RB.

The peak rate of UE $k$ when associated with RB $n$ on cell $i$ is given by:

$$
\rho_{k, i, n}=\log \left(1+\frac{\pi_{i, n} G_{k, i, n}}{N_{0}+\sum_{i^{\prime} \neq i} \pi_{i^{\prime}, n} G_{k, i^{\prime}, n}}\right) .
$$

Then, the mean rate of UE $k$ is given by:

$$
\begin{aligned}
& \sum_{n \in \mathcal{N}}\left(\theta_{k, n} \cdot \rho_{k, i, n}\right)= \\
& \sum_{n \in \mathcal{N}}\left(\theta_{k, n} \cdot \log \left(1+\frac{\pi_{i, n} G_{k, i, n}}{N_{0}+\sum_{i^{\prime} \neq i} \pi_{i^{\prime}, n} G_{k, i^{\prime}, n}}\right)\right) .
\end{aligned}
$$

Our centralized multi-cell joint resource and power allocation problem seeks rate maximization in a proportional fair manner. We make use of the logarithmic function that is intimately associated with the concept of proportional fairness [27]. Our problem is formulated in the following:

$$
\begin{array}{ll}
\underset{\boldsymbol{\theta}, \boldsymbol{\pi}}{\operatorname{maximize}} & \eta= \\
\sum_{i \in \mathcal{I}} \sum_{k \in \mathcal{K}(i)} \log \left(\sum_{n \in \mathcal{N}} \theta_{k, n} \cdot \log \left(1+\frac{\pi_{i, n} G_{k, i, n}}{N_{0}+\sum_{i^{\prime} \neq i} \pi_{i^{\prime}, n} G_{k, i^{\prime}, n}}\right)\right) \\
\text { subject to } \quad \sum_{k \in \mathcal{K}(i)} \theta_{k, n} \leq 1, \forall n \in \mathcal{N}, \\
& \sum_{n \in \mathcal{N}} \theta_{k, n} \leq 1, \forall k \in \mathcal{K}(i), \\
& \sum_{n \in \mathcal{N}} \pi_{i, n} \leq P_{\max }, \forall i \in \mathcal{I}, \\
& \pi_{i, n} \geq \pi_{\min }, \forall i \in \mathcal{I}, \forall n \in \mathcal{N}, \\
& 0 \leq \theta_{k, n} \leq 1, \forall k \in \mathcal{K}(i), \forall n \in \mathcal{N} .
\end{array}
$$

The objective function $\eta$ ensures a proportional fair rate for all UEs in the network. Constraints (4b) ensure that an RB is used at most $100 \%$ of the time, 
and constraints (4c) ensure that a UE shares its time on the available RBs. Constraints (4d) guarantee that the total downlink transmission power allocated to the available RBs does not exceed the maximum transmission power $P_{\max }$ for each cell $i$, and constraints (4e) represent the minimum power constraint of the transmit power allocated to each RB. In fact, the power allocated to each RB is larger than a predefined value denoted $\pi_{m i n}$, and the transmit power of cell $i$ is lower than $P_{\max }$. In practice, these bounds are related to hardware limitations.

$\theta_{k, n}, \forall k \in \mathcal{K}, \forall n \in \mathcal{N}$, and $\pi_{i, n}, \forall i \in \mathcal{I}, \forall n \in \mathcal{N}$ are the optimization variables of the joint resource and power allocation problem.

In order to reduce the complexity of the joint resource and power allocation problem (4), we prove that this problem is separable into two independent problems: a resource allocation problem and a power allocation problem. In fact, maximizing the objective function of problem (4) is achieved by maximizing the following term:

$$
\sum_{i \in \mathcal{I}} \sum_{k \in \mathcal{K}(i)} \sum_{n \in \mathcal{N}}\left(\log \left(\theta_{k, n}\right)+\log \left(\rho_{k, i, n}\right)\right) .
$$

The proof of this hypothesis is given in Appendix I.

\section{Problem Decomposition}

We tackle ICIC as an optimization problem, where we intend to maximize the mean rate of UEs in a multiuser OFDMA system. We consider a system of $I$ cells, having $K(i)$ UEs per cell $i$. According to (5), and due to the absence of binding constraints, the optimization problem (4) is linearly separable into two independent problems: a power allocation problem and a resource allocation problem.

In the first problem, the optimization variable $\boldsymbol{\pi}$ is considered, and the problem is formulated as follows: 


$$
\begin{array}{ll}
\underset{\pi}{\operatorname{maximize}} & \eta_{1}= \\
\qquad \sum_{i \in \mathcal{I}} \sum_{k \in \mathcal{K}(i)} \sum_{n \in \mathcal{N}} \log \left(\log \left(1+\frac{\pi_{i, n} G_{k, i, n}}{N_{0}+\sum_{i^{\prime} \neq i} \pi_{i^{\prime}, n} G_{k, i^{\prime}, n}}\right)\right) \\
\text { subject to } & \sum_{n \in \mathcal{N}} \pi_{i, n} \leq P_{\text {max }}, \forall i \in \mathcal{I}, \\
& \pi_{i, n} \geq \pi_{\text {min }}, \forall i \in \mathcal{I}, \forall n \in \mathcal{N} .
\end{array}
$$

Problem (6) consists in finding the optimal power allocation. However, it is not a convex optimization problem as formulated in (6). In the following, we optimization problem as follows:

$$
\begin{array}{ll}
\underset{\boldsymbol{\rho}, \boldsymbol{\pi}}{\operatorname{maximize}} & \eta_{1}=\sum_{i \in \mathcal{I}} \sum_{k \in \mathcal{K}(i)} \sum_{n \in \mathcal{N}} \log \left(\rho_{k, i, n}\right) \\
\text { subject to } & \rho_{k, i, n} \leq \log \left(1+\frac{\pi_{i, n} G_{k, i, n}}{N_{0}+\sum_{i^{\prime} \neq i} \pi_{i^{\prime}, n} G_{k, i^{\prime}, n}}\right), \\
& \forall i \in \mathcal{I}, \forall k \in \mathcal{K}(i), \forall n \in \mathcal{N}, \\
& \sum_{n \in \mathcal{N}} \pi_{i, n} \leq P_{\text {max }}, \forall i \in \mathcal{I}, \\
& \pi_{i, n} \geq \pi_{\text {min }}, \forall i \in \mathcal{I}, \forall n \in \mathcal{N} .
\end{array}
$$

Let us consider the following variable change:

$$
\begin{aligned}
& \widehat{\rho}_{k, i, n}=\log \left(\exp \left(\rho_{k, i, n}\right)-1\right), \forall i \in \mathcal{I}, \forall k \in \mathcal{K}(i), \forall n \in \mathcal{N}, \\
& \widehat{\pi}_{i, n}=\log \left(\pi_{i, n}\right), \forall i \in \mathcal{I}, \forall n \in \mathcal{N} .
\end{aligned}
$$

Hence, the original variables are given by:

$$
\begin{aligned}
& \rho_{k, i, n}=\log \left(\exp \left(\widehat{\rho}_{k, i, n}\right)+1\right), \forall i \in \mathcal{I}, \forall k \in \mathcal{K}(i), \forall n \in \mathcal{N}, \\
& \pi_{i, n}=\exp \left(\widehat{\pi}_{i, n}\right), \forall i \in \mathcal{I}, \forall n \in \mathcal{N} .
\end{aligned}
$$

To show that the optimization problem (7) is a convex optimization problem, we need to show that the objective function is concave and the inequality 
peak rate constraints $(7 \mathrm{~b})$, they can be written as:

$$
\begin{aligned}
& \rho_{k, i, n} \leq \log \left(1+\frac{\pi_{i, n} G_{k, i, n}}{N_{0}+\sum_{i^{\prime} \neq i} \pi_{i^{\prime}, n} G_{k, i^{\prime}, n}}\right), \\
& \forall i \in \mathcal{I}, \forall k \in \mathcal{K}(i), \forall n \in \mathcal{N} \\
\Rightarrow & \log \left(\exp \left(\widehat{\rho}_{k, i, n}\right)+1\right) \leq \log \left(1+\frac{\exp \left(\widehat{\pi}_{i, n}\right) G_{k, i, n}}{N_{0}+\sum_{i^{\prime} \neq i} \exp \left(\widehat{\pi}_{i^{\prime}, n}\right) G_{k, i^{\prime}, n}}\right) \\
\Rightarrow & \exp \left(\widehat{\rho}_{k . i . n}\right)+1 \leq 1+\frac{\exp \left(\widehat{\pi}_{i, n}\right) G_{k, i, n}}{N_{0}+\sum_{i^{\prime} \neq i} \exp \left(\widehat{\pi}_{i^{\prime}, n}\right) G_{k, i^{\prime}, n}} \\
\Rightarrow & \frac{\exp \left(\widehat{\rho}_{k . i . n}\right) \cdot\left(N_{0}+\sum_{i^{\prime} \neq i} \exp \left(\widehat{\pi}_{i^{\prime}, n}\right) G_{k, i^{\prime}, n}\right)}{\exp \left(\widehat{\pi}_{i, n}\right) G_{k, i, n}} \leq 1 \\
\Rightarrow & \log \left(\exp \left(\widehat{\rho}_{k . i . n}-\widehat{\pi}_{i, n}\right) \frac{N_{0}}{G_{k, i, n}}+\sum_{i^{\prime} \neq i} \exp \left(\widehat{\rho}_{k i . n}+\widehat{\pi}_{i^{\prime}, n}-\widehat{\pi}_{i, n}\right) \frac{G_{k, i^{\prime}, n}}{G_{k, i, n}}\right) \leq 0, \\
& \forall i \in \mathcal{I}, \forall k \in \mathcal{K}(i), \forall n \in \mathcal{N} .
\end{aligned}
$$

These constraints are the logarithmic of the sum of exponential functions. Thus, they are convex functions [28]. When we apply the variable change on power constraints $(7 \mathrm{c})$, we get:

$$
\begin{aligned}
& \sum_{n \in \mathcal{N}} \pi_{i, n} \leq P_{\text {max }}, \forall i \in \mathcal{I} \\
\Rightarrow & \log \left(\sum_{n \in \mathcal{N}} \exp \left(\widehat{\pi}_{i, n}\right)\right)-\log \left(P_{\text {max }}\right) \leq 0, \forall i \in \mathcal{I} .
\end{aligned}
$$

Since $\log \left(\sum \exp \right)$ is convex [28], the constraints at hand are therefore convex. Using the variable change, the power allocation problem (7) can be written as follows:

$$
\underset{\widehat{\boldsymbol{\rho}}, \boldsymbol{\pi}}{\operatorname{maximize}} \quad \eta_{1}=\sum_{i \in \mathcal{I}} \sum_{k \in \mathcal{K}(i)} \sum_{n \in \mathcal{N}} \log \left(\log \left(\exp \left(\widehat{\rho}_{k, i, n}\right)+1\right)\right)
$$

subject to

$$
\begin{aligned}
\log \left(\exp \left(\widehat{\rho}_{k . i . n}-\widehat{\pi}_{i, n}\right) \frac{N_{0}}{G_{k, i, n}}+\sum_{i^{\prime} \neq i} \exp \left(\widehat{\rho}_{k . i . n}+\widehat{\pi}_{i^{\prime}, n}-\widehat{\pi}_{i, n}\right) \frac{G_{k, i^{\prime}, n}}{G_{k, i, n}}\right) \leq 0 \\
\forall i \in \mathcal{I}, \forall k \in \mathcal{K}(i), \forall n \in \mathcal{N} \\
\\
\log \left(\sum_{n \in \mathcal{N}} \exp \left(\widehat{\pi}_{i, n}\right)\right)-\log \left(P_{\text {max }}\right) \leq 0, \forall i \in \mathcal{I} \\
\widehat{\pi}_{i, n} \geq \log \left(\pi_{\text {min }}\right), \forall i \in \mathcal{I}, \forall n \in \mathcal{N}
\end{aligned}
$$


The objective function of problem (10) is concave in $\widehat{\boldsymbol{\rho}}$ and $\widehat{\boldsymbol{\pi}}$, and constraints (10b), (10c), and (10d) are convex functions. Thus, the power allocation problem is a convex optimization problem.

\subsection{Centralized Resource Allocation Problem}

The optimization variable $\boldsymbol{\theta}$ is considered in the second optimization problem that is given in the following:

$$
\begin{array}{ll}
\underset{\boldsymbol{\theta}}{\operatorname{maximize}} & \eta_{2}=\sum_{i \in \mathcal{I}} \sum_{k \in \mathcal{K}(i)} \sum_{n \in \mathcal{N}} \log \left(\theta_{k, n}\right) \\
\text { subject to } & \sum_{k \in \mathcal{K}(i)} \theta_{k, n} \leq 1, \forall n \in \mathcal{N}, \\
& \sum_{n \in \mathcal{N}} \theta_{k, n} \leq 1, \forall k \in \mathcal{K}(i), \\
& 0 \leq \theta_{k, n} \leq 1, \forall k \in \mathcal{K}(i), \forall n \in \mathcal{N} .
\end{array}
$$

As demonstrated for the power allocation problem (6), we prove that problem (11) is indeed a convex optimization problem in $\boldsymbol{\theta}$. The objective function (11a) of the resource allocation problem (11) is concave in $\boldsymbol{\theta}$, since the log function is concave for $\boldsymbol{\theta} \in] 0 ; 1]$. Moreover, constraints (11b), (11c), and (11d) are linear and separable constraints. Hence, the resource allocation problem (11) is a convex optimization problem, and it is separable into $\mathcal{I}$ subproblems. For each cell $i$, the $i$ th optimization problem is written as follows:

$$
\begin{array}{ll}
\underset{\boldsymbol{\theta}}{\operatorname{maximize}} & \left(\eta_{2}\right)_{i}=\sum_{k \in \mathcal{K}(i)} \sum_{n \in \mathcal{N}} \log \left(\theta_{k, n}\right) \\
\text { subject to } & \sum_{k \in \mathcal{K}(i)} \theta_{k, n} \leq 1, \forall n \in \mathcal{N}, \\
& \sum_{n \in \mathcal{N}} \theta_{k, n} \leq 1, \forall k \in \mathcal{K}(i), \\
& 0 \leq \theta_{k, n} \leq 1, \forall k \in \mathcal{K}(i), \forall n \in \mathcal{N} .
\end{array}
$$

\section{Centralized Multi-Cell Resource and Power Allocation}

As stated in the previous section and proven in Appendix I, the joint resource and power allocation problem (4) is separable into two independent convex 
optimization problems: a power allocation problem, and a resource allocation problem. In this section, we solve the resource and power allocation problems using Lagrange duality theory and subgradient projection method.

\subsection{Solving the Centralized Power Allocation Problem}

\subsubsection{Lagrange-Based Method}

Since the power allocation problem (10) is a convex optimization problem, we

can make use of Lagrange duality properties, which also lead to decomposability structures [29]. Lagrange duality theory links the original problem, or primal problem, with a dual maximization problem. The Lagrangian of problem (10) is given as follows:

$$
\begin{aligned}
L(\widehat{\boldsymbol{\rho}}, \widehat{\boldsymbol{\pi}}, \boldsymbol{\lambda}, \boldsymbol{\nu})=\sum_{i \in \mathcal{I}} \sum_{k \in \mathcal{K}(i)} \sum_{n \in \mathcal{N}} \log \left(\log \left(\exp \left(\widehat{\rho}_{k, i, n}\right)+1\right)\right) \\
\quad-\sum_{i \in \mathcal{I}} \sum_{k \in \mathcal{K}(i)} \sum_{n \in \mathcal{N}} \lambda_{k, i, n}\left(\operatorname { l o g } \left(\exp \left(\widehat{\rho}_{k, i . n}-\widehat{\pi}_{i, n}\right) \frac{N_{0}}{G_{k, i, n}}\right.\right. \\
\left.\left.+\sum_{\substack{i^{\prime} \in \mathcal{N} \\
i^{\prime} \neq i}} \exp \left(\widehat{\rho}_{k, i . n}+\widehat{\pi}_{i^{\prime}, n}-\widehat{\pi}_{i, n}\right) \frac{G_{k, i^{\prime}, n}}{G_{k, i, n}}\right)\right) \\
-\sum_{i \in \mathcal{I}} \nu_{i}\left(\log \left(\sum_{n \in \mathcal{N}} \exp \left(\widehat{\pi}_{i, n}\right)\right)-\log \left(P_{\text {max }}\right)\right)
\end{aligned}
$$

The optimization variables $\widehat{\boldsymbol{\rho}}$ and $\widehat{\boldsymbol{\pi}}$ are called the primal variables. $\lambda_{k, i, n}$ and $\nu_{i}$ are the dual variables associated with the $(k, i, n)$ th inequality constraint (10b) and with the $i$ th inequality constraint (10c), respectively.

After relaxing the coupling constraints (10b) and (10c), the optimization problem separates into two levels of optimization: lower level and higher level. At the lower level, $L(\widehat{\boldsymbol{\rho}}, \widehat{\boldsymbol{\pi}}, \boldsymbol{\lambda}, \boldsymbol{\nu})$ is the objective function to be maximized. $\widehat{\rho}_{k, i, n}$ and $\widehat{\pi}_{i, n}$ are the optimization variables to be found. At the higher level, we have the master dual problem in charge of updating the dual variables $\boldsymbol{\lambda}$ and $\boldsymbol{\nu}$ by 
solving the dual problem:

$$
\begin{array}{ll}
\underset{\boldsymbol{\lambda}, \boldsymbol{\nu}}{\operatorname{minimize}} & \max _{\widehat{\boldsymbol{\rho}}, \hat{\boldsymbol{\pi}}}(L(\widehat{\boldsymbol{\rho}}, \widehat{\boldsymbol{\pi}}, \boldsymbol{\lambda}, \boldsymbol{\nu})) \\
\text { subject to } & \boldsymbol{\lambda} \geq 0, \\
& \boldsymbol{\nu} \geq 0 .
\end{array}
$$

In order to solve the primal optimization problem (lower level of optimization), we use the subgradient projection method. It starts with some initial feasible values of $\widehat{\rho}_{k, i, n}$ and $\widehat{\pi}_{i, n}$ that satisfy the constraints (10d). Then, the next iteration is generated by taking a step along the subgradient direction of $\widehat{\rho}_{k, i, n}$ and $\widehat{\pi}_{i, n}$. For the primal optimization variables, iterations of the subgradient projection are given by:

$$
\begin{aligned}
& \widehat{\rho}_{k, i, n}(t+1)=\widehat{\rho}_{k, i, n}(t)+\delta(t) \times \frac{\partial L}{\partial \widehat{\rho}_{k, i, n}}, \\
& \forall k \in \mathcal{K}(i), \forall i \in \mathcal{I}, \forall n \in \mathcal{N}, \\
& \widehat{\pi}_{i, n}(t+1)=\widehat{\pi}_{i, n}(t)+\delta(t) \times \frac{\partial L}{\partial \widehat{\pi}_{i, n}}, \forall i \in \mathcal{I}, \forall n \in \mathcal{N} .
\end{aligned}
$$

The scalar $\delta(t)$ is a step size that guarantees the convergence of the primal optimization problem [29]. The partial derivatives of the objective function $L(\widehat{\boldsymbol{\rho}}, \widehat{\boldsymbol{\pi}}, \boldsymbol{\lambda}, \boldsymbol{\nu})$ with respect to $\widehat{\rho}_{k, i, n}$ and $\widehat{\pi}_{i, n}$, are given in the following:

$$
\begin{aligned}
\frac{\partial L}{\partial \widehat{\rho}_{k, i, n}} & =\frac{\exp \left(\widehat{\rho}_{k, i, n}\right)}{\left(\exp \left(\widehat{\rho}_{k, i, n}\right)+1\right) \log \left(\exp \left(\widehat{\rho}_{k, i, n}\right)+1\right)}-\lambda_{k, i, n}, \\
\forall k & \in \mathcal{K}(i), \forall i \in \mathcal{I}, \forall n \in \mathcal{N}, \\
\frac{\partial L}{\partial \widehat{\pi}_{i, n}} & =\sum_{k \in \mathcal{K}(i)} \lambda_{k, i, n}-\nu_{i} \frac{\exp \left(\widehat{\pi}_{i, n}\right)}{\sum_{n \in \mathcal{N}} \exp \left(\widehat{\pi}_{i, n}\right)}, \forall i \in \mathcal{I}, \forall n \in \mathcal{N} .
\end{aligned}
$$

The dual function $g(\boldsymbol{\lambda}, \boldsymbol{\nu})=\max _{\widehat{\boldsymbol{\rho}}, \hat{\boldsymbol{\pi}}}(L(\widehat{\boldsymbol{\rho}}, \widehat{\boldsymbol{\pi}}, \boldsymbol{\lambda}, \boldsymbol{\nu}))$ is differentiable. Thus, at the higher optimization level, the master dual problem (14) can be solved using 
the following gradient method:

$$
\begin{aligned}
& \lambda_{k, i, n}(t+1)=\lambda_{k, i, n}(t)+\delta(t)\left(\operatorname { l o g } \left(\exp \left(\widehat{\rho}_{k . i . n}^{\star}-\widehat{\pi}_{i, n}^{\star}\right) \frac{N_{0}}{G_{k, i, n}}\right.\right. \\
&\left.\left.+\sum_{\substack{i^{\prime} \in \mathcal{N} \\
i^{\prime} \neq i}} \exp \left(\widehat{\rho}_{k . i . n}^{\star}+\widehat{\pi}_{i^{\prime}, n}^{\star}-\widehat{\pi}_{i, n}^{\star}\right) \frac{G_{k, i^{\prime}, n}}{G_{k, i, n}}\right)\right), \\
& \forall k \in \mathcal{K}(i), \forall i \in \mathcal{I}, \forall n \in \mathcal{N}, \\
& \nu_{i}(t+1)=\nu_{i}(t)+\delta(t)\left(\log \left(\sum_{n \in \mathcal{N}} \exp \left(\widehat{\pi}_{i, n}^{\star}\right)\right)-\log \left(P_{\max }\right)\right), \\
& \forall i \in \mathcal{I}, \forall n \in \mathcal{N},
\end{aligned}
$$

where $t$ is the iteration index, and $\delta(t)$ is the step size at iteration $t$. Appropriate choice of the step size [30] leads to convergence of the dual algorithm. $\widehat{\pi}_{i, n}^{\star}$ and $\widehat{\rho}_{k, i, n}^{\star}$ denote the solution to the primal optimization problem. When $t \rightarrow \infty$ the dual variables $\boldsymbol{\lambda}(t)$ and $\boldsymbol{\nu}(t)$ converge to the dual optimal $\boldsymbol{\lambda}^{*}$ and $\boldsymbol{\nu}^{*}$, respectively. The difference between the optimal primal objective and the optimal dual objective, called duality gap, reduces to zero at optimality, since the problem (10) is convex and the KKT conditions are satisfied. We define $\Delta \widehat{\boldsymbol{\rho}}, \Delta \widehat{\boldsymbol{\pi}}, \Delta \boldsymbol{\lambda}$, and $\Delta \boldsymbol{\nu}$ as the differences between the optimization variables obtained at the current iteration and their values at the previous iteration. They are given by:

$$
\begin{aligned}
\Delta \widehat{\boldsymbol{\rho}}(t+1) & =\|\widehat{\boldsymbol{\rho}}(t+1)-\widehat{\boldsymbol{\rho}}(t)\|, \\
\Delta \widehat{\boldsymbol{\pi}}(t+1) & =\|\widehat{\boldsymbol{\pi}}(t+1)-\widehat{\boldsymbol{\pi}}(t)\|, \\
\Delta \boldsymbol{\lambda}(t+1) & =\|\boldsymbol{\lambda}(t+1)-\boldsymbol{\lambda}(t)\|, \\
\Delta \boldsymbol{\nu}(t+1) & =\|\boldsymbol{\nu}(t+1)-\boldsymbol{\nu}(t)\| .
\end{aligned}
$$

\subsubsection{Iterative Power Allocation Algorithm}

The procedure for solving the centralized power allocation problem is described in Algorithm 1. Initially, the primal optimization variables $\widehat{\rho}_{k, i, n}$ and $\widehat{\pi}_{i, n}$ as well as the dual variables $\lambda_{k, i, n}$ and $\nu_{i}$ start with some initial feasible values. $t, t_{\text {primal }}$, and $t_{d u a l}$ denote the number of rounds required for the centralized 


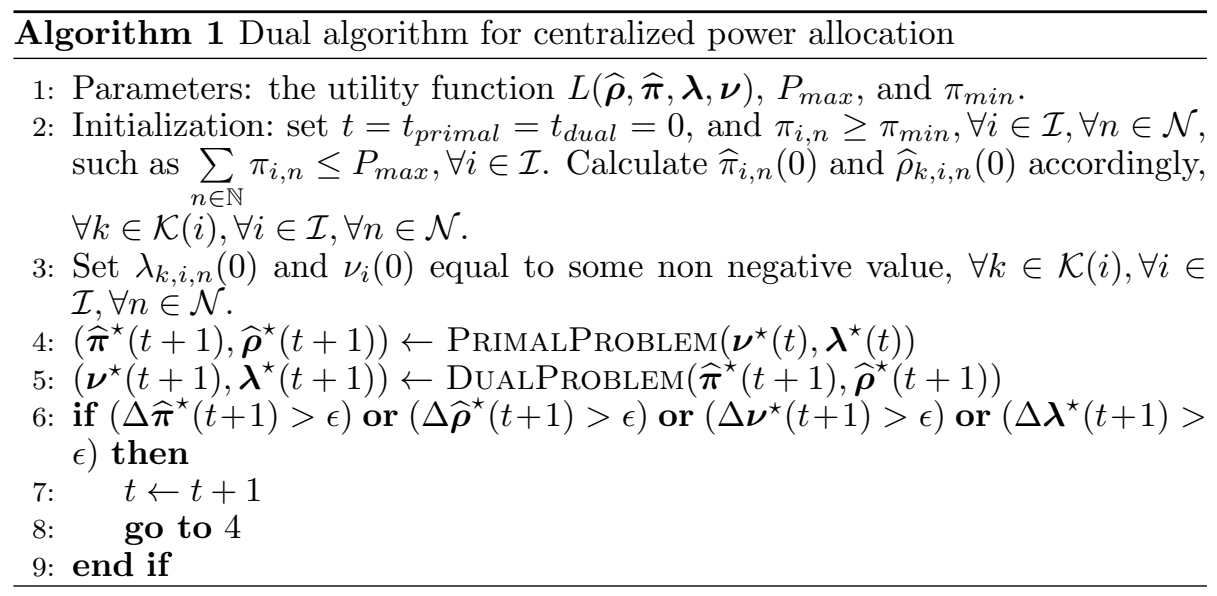

power allocation problem to converge, the number of iterations for the primal problem, and the number of iterations for the dual problem, respectively. At each round $t$, we start by updating the primal optimization variables, using the PrimalProblem function given in Algorithm 2. The solution to the primal optimization problem at the current round $t$ is denoted by $\widehat{\pi}_{i, n}^{\star}(t+1)$ and $\widehat{\rho}_{k, i, n}^{\star}(t+1)$. The PrimalProblem function updates $\widehat{\pi}_{i, n}\left(t_{\text {primal }}+1\right)$ and $\widehat{\rho}_{k, i, n}\left(t_{\text {primal }}+1\right)$, and increments $t_{\text {primal }}$ until $\Delta \widehat{\boldsymbol{\pi}}\left(t_{\text {primal }}+1\right)$ and $\Delta \widehat{\boldsymbol{\rho}}\left(t_{\text {primal }}+\right.$ 1) become less than $\epsilon$.

Then, the solution to the dual optimization problem at the current round $t$, denoted by $\nu_{i}^{\star}(t+1)$ and $\lambda_{k, i, n}^{\star}(t+1)$ is calculated using the DualProblem function given in Algorithm 3. $\nu_{i}$ and $\lambda_{k, i, n}$ are updated using the obtained primal solution $\widehat{\pi}_{i, n}^{\star}(t+1)$ and $\widehat{\rho}_{k, i, n}^{\star}(t+1)$, until $\Delta \boldsymbol{\nu}\left(t_{\text {dual }}+1\right)$ and $\Delta \boldsymbol{\lambda}\left(t_{\text {dual }}+1\right)$ become less than $\epsilon$. An additional round of calculations is performed, and $t$ is incremented as long as $\Delta \widehat{\boldsymbol{\pi}}^{\star}(t+1)$ or $\Delta \widehat{\boldsymbol{\pi}}^{\star}(t+1)$ or $\Delta \boldsymbol{\nu}^{\star}(t+1)$ or $\Delta \boldsymbol{\lambda}^{\star}(t+1)$ is greater than $\epsilon$. Otherwise, the obtained solution at the current round is the optimal solution to the centralized power allocation problem.

\subsection{Solving the Resource Allocation Problem}

In this subsection, we search for the optimal solution to the resource allocation problem (12). For each cell $i$, the problem (12) is a convex optimization problem, as proven previously. 


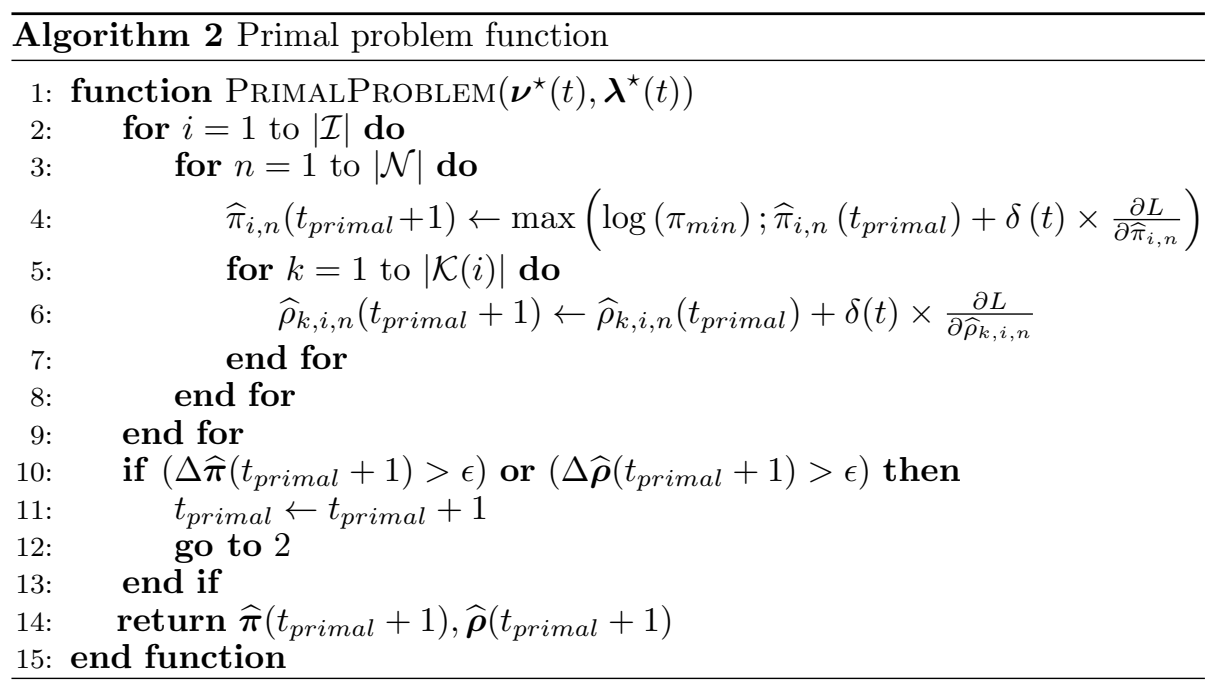

Theorem 5.1. For each cell $i$, the optimal solution to the resource allocation problem (12) is given by:

$$
\theta_{k, n}=\frac{1}{\max (|\mathcal{K}(i)|,|\mathcal{N}|)}, \forall k \in \mathcal{K}(i), \forall n \in \mathcal{N}
$$

The proof of Theorem 5.1 is given in Appendix II. When the number of active UEs is less than the number of available resources, $\theta_{k, n}=\frac{1}{|\mathcal{N}|}, \forall k \in$ ${ }_{275} \mathcal{K}(i), \forall n \in \mathcal{N}$. Thus, the available resources are not fully used over time, and each UE is permanently served. Otherwise, when $|\mathcal{K}(i)|>|\mathcal{N}|$, the optimal solution is: $\theta_{k, n}=\frac{1}{|\mathcal{K}(i)|}, \forall k \in \mathcal{K}(i), \forall n \in \mathcal{N}$. In this case, each RB is fully used over time, while UEs are not permanently served over time.

\section{Decentralized Resource and Power Allocation}

\subsection{Problem Formulation and Decomposition}

We have shown that the power allocation problem can be solved optimally in a centralized fashion. The centralized approach is the reference approach for performance comparison, since it finds the optimal resource and power allocation for all the active users. Nevertheless, the computational complexity of the 


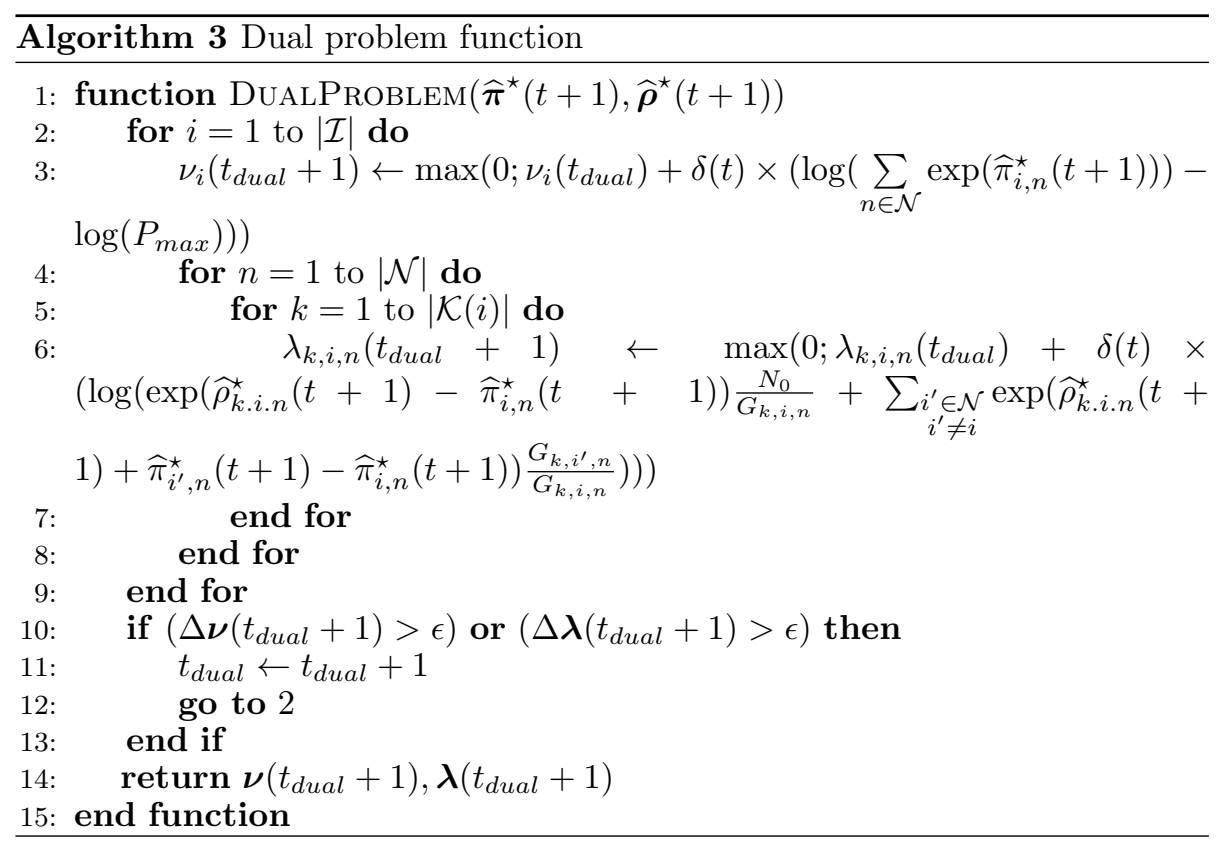

centralized approach motivates the introduction of low-complexity decentralized approaches. In this section we investigate the decentralized resource and power allocation approach. Base stations of the LTE/LTE-A networks are autonomous entities, and each cell performs resource and power allocation independently of the other cells. Each cell $i$ maximizes its own utility function, which is given by:

$$
\begin{aligned}
& \sum_{k \in \mathcal{K}(i)} \sum_{n \in \mathcal{N}} \log \left(\theta_{k, n} \cdot \log \left(1+\frac{\pi_{i, n} G_{k, i, n}}{N_{0}+\sum_{i^{\prime} \neq i} \pi_{i^{\prime}, n} G_{k, i^{\prime}, n}}\right)\right)= \\
& \quad \sum_{k \in \mathcal{K}(i)} \sum_{n \in \mathcal{N}} \log \left(\theta_{k, n}\right)+ \\
& \quad \sum_{k \in \mathcal{K}(i)} \sum_{n \in \mathcal{N}} \log \left(\log \left(1+\frac{\pi_{i, n} G_{k, i, n}}{N_{0}+\sum_{i^{\prime} \neq i} \pi_{i^{\prime}, n} G_{k, i^{\prime}, n}}\right)\right) .
\end{aligned}
$$

The decentralized joint resource and power allocation problem is separable into two independent problems: a resource allocation problem and a power allocation problem. The resource allocation problem is solved in a distributed manner as proven in the previous section. We propose a decentralized power allocation 
285

295

approach based on game theory, where the cells are the decision makers or players of the game. We define a multi-player game $\mathcal{G}$ between the $|\mathcal{I}|$ cells. The cells are assumed to make their decisions without knowing the decisions of each other.

The formulation of this non-cooperative game $G=\langle\mathcal{I}, S, U\rangle$ can be described as follows:

- A finite set of cells $\mathcal{I}=(1, \ldots,|\mathcal{I}|)$.

- For each cell $i$, the space of pure strategies is $S_{i}$ given by what follows: $S_{i}=\left\{\pi_{i} \in R^{|\mathcal{N}|}\right.$ such as $\pi_{i, n} \geq \pi_{m i n}, \forall i \in \mathcal{I}, \forall n \in \mathcal{N}$, and $\sum_{n \in \mathcal{N}} \pi_{i, n} \leq$ $\left.P_{\max }, \forall i \in \mathcal{I}\right\}$.

An action of a cell $i$ is the amount of power $\pi_{i, n}$ allocated to the RB $n$, and the strategy chosen by cell $i$ is then $\pi_{i}=\left(\pi_{i, 1}, \ldots, \pi_{i, N}\right)$. A strategy profile $\pi=\left(\pi_{1}, \ldots, \pi_{|\mathcal{I}|}\right)$ specifies the strategies of all players and $S=S_{1} \times \ldots \times S_{|\mathcal{I}|}$ is the set of all strategies.

- A set of utility functions $U=\left(U_{1}(\pi), U_{2}(\pi), \ldots, U_{I}(\pi)\right)$ that quantify players' utility for a given strategy profile $\pi$, where a given utility $U_{i}$ for cell $i$ is such as:

$$
U_{i}=\sum_{k \in \mathcal{K}(i)} \sum_{n \in \mathcal{N}} \log \left(\log \left(1+\frac{\pi_{i, n} G_{k, i, n}}{N_{0}+\sum_{i^{\prime} \neq i} \pi_{i^{\prime}, n} G_{k, i^{\prime}, n}}\right)\right) .
$$

For every $i, U_{i}$ is concave w.r.t. $\pi_{i}$ and continuous w.r.t. $\pi_{l}, l \neq i$. Hence, a soo Nash Equilibrium (NE) exists [31]. We note that the objective function $\eta_{1}$ of the centralized power allocation problem (10) is equivalent to the sum of the utility functions $U_{i}$ of the $I$ cells.

\subsection{Super-Modular Games}

Super-modular games exhibit strategic complementarity i.e., the marginal utility for a player in playing a higher strategy increases when the opponents also play higher strategy [32]. These games encompass many applied models, and 
they are characterized by the existence of pure strategy NE. Before presenting the properties of a super-modular game, we list first the following definition:

Definition 6.1. If $U_{i}$ is twice differentiable, it is said to be super-modular if:

$$
\frac{\partial U_{i}}{\partial \pi_{l} \partial \pi_{i}} \geq 0, \forall l \in \mathcal{I}-\{i\}, \forall \pi_{i} \in S_{i}
$$

According to [33], a game is super-modular if $\forall i \in \mathcal{I}$ :

1. The strategy space $S_{i}$ is a compact sublattice of $\mathbb{R}^{N}$.

2. The utility function $U_{i}$ is super-modular.

In $[33,34]$, proof is given for the following result in a super-modular game:

- If we start with a feasible policy, then the sequence of best responses monotonically converges to an equilibrium: it monotonically increases in all components in the case of maximizing in a super-modular game.

Proposition 6.2. The game $\mathcal{G}$ is a super-modular game.

The proof of this proposition is given in Appendix III.

To attain the NE of the game, we implement a best response algorithm where in each round $t$, cell $i$ strives to find, in parallel for every RB $n \in$ $\mathcal{N}$, the following optimal power level as a response to $\pi_{-i}(t-1): \pi_{i}^{*}(t)=$ $\arg \max _{\pi_{i}} U_{i}\left(\pi_{i}, \pi_{-i}\right)$, s.t. $\pi_{i}^{*} \in S_{i}$.

The resulting optimization problem for each cell $i$ is as follows:

$$
\begin{aligned}
\underset{\pi_{i}}{\operatorname{maximize}} & U_{i} \\
\text { subject to: } & \sum_{n \in \mathcal{N}} \pi_{i, n} \leq P_{\text {max }}, \\
& \pi_{i, n} \geq \pi_{\text {min }}, \forall n \in \mathcal{N} .
\end{aligned}
$$

\subsection{Solving the Decentralized Power Allocation Problem}

We use the subgradient projection method to solve the decentralized power allocation problem (22). It is an iterative method that starts with some initial 
feasible vector $\boldsymbol{\pi}_{i}$ that satisfies constraints (22b) and (22c), and generates the next iteration by taking a step along the subgradient direction of $U_{i}$ at $\boldsymbol{\pi}_{i}$. For each cell $i$, iterations of the subgradient projection are given by:

$$
\pi_{i, n}(t+1)=\pi_{i, n}(t)+\delta(t) \times \frac{\partial U_{i}}{\partial \pi_{i, n}}, \forall n \in \mathcal{N},
$$

where the partial derivative of the objective function $U_{i}$ with respect to $\pi_{i, n}$ is given by:

$$
\begin{aligned}
\frac{\partial U_{i}}{\partial \pi_{i, n}} & =\sum_{k \in \mathcal{K}(i)} \frac{G_{k, i, n}}{\left(N_{0}+F_{i, n}\right)\left(1+\frac{\pi_{i, n} G_{k, i, n}}{N_{0}+F_{i, n}}\right) \log \left(1+\frac{\pi_{i, n} G_{k, i, n}}{N_{0}+F_{i, n}}\right)}, \\
F_{i, n} & =\sum_{\substack{i^{\prime} \in \mathcal{I} \\
i^{\prime} \neq i}} \pi_{i^{\prime}, n} G_{k, i^{\prime}, n}, \forall n \in \mathcal{N} .
\end{aligned}
$$

The scalar $\delta(t)>0$ is a small step size $(e . g ., \delta(t)=0.001)$ chosen appropriately [30] to guarantee the convergence of the decentralized power allocation problem (22). Before updating the variables $\pi_{i, n}(t+1)$, we make sure that $\pi_{i, n}(t+1) \geq \pi_{\min }$ in order to satisfy the constraints (22c). Moreover, if constraints $(22 \mathrm{~b})$ are not satisfied, we perform a projection on the feasible set $P_{\max }$, which is straightforward for a simplex [35]. Then, we calculate the power difference $\Delta \pi_{i}$, which is the difference between the power allocation vectors of the current and the previous iterations. It is given by:

$$
\Delta \pi_{i}(t+1)=\left\|\pi_{i}(t+1)-\pi_{i}(t)\right\|
$$

As described in Algorithm 4, each cell $i$ calculates $\pi_{i, n}\left(t_{i}+1\right), \forall n \in \mathcal{N}$, where $t_{i}$ is the iteration number for cell $i$. The obtained power values are updated in accordance with the constraints (22b) and (22c). This procedure is repeated and the number of iterations $t_{i}$ is incremented until $\Delta \pi_{i}\left(t_{i}+1\right)$ becomes less than $\epsilon$. The number of rounds required for all the cells to converge is denoted by $t$. An additional round of power calculation is performed for all the cells and $t$ is incremented as long as $\Delta \pi^{*}(t+1)>\epsilon$, where $\pi^{*}(t)$ is the 


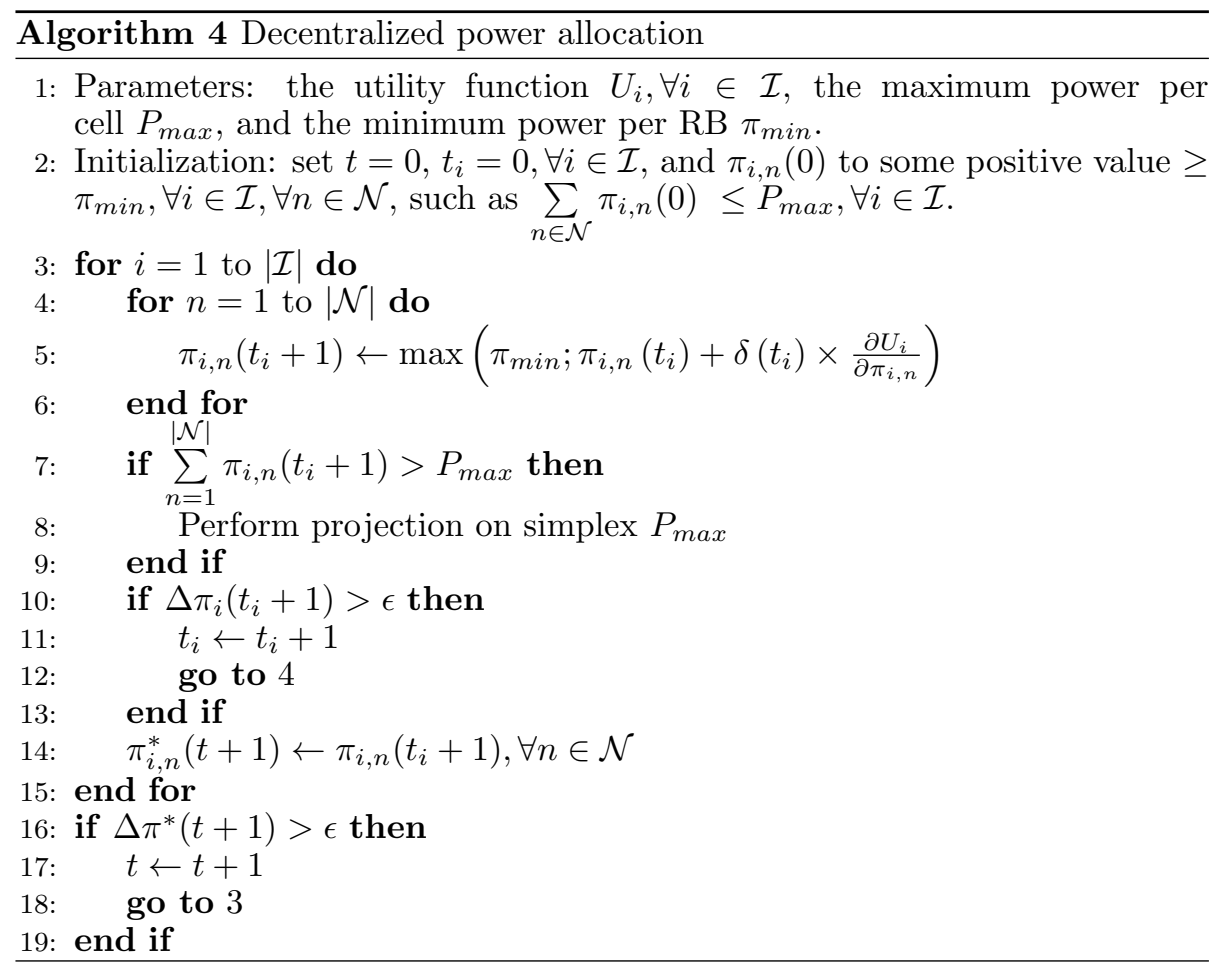

330 power allocation vector obtained at the end of round $t$.

\section{Performance Evaluation}

In this section, we evaluate the convergence and the performance of the proposed centralized joint resource and power allocation problem, and the decentralized power allocation approach.

\subsection{Centralized Resource and Power Allocation}

To verify the convergence of the centralized solution, we consider a multiuser OFDMA network, such as LTE/LTE-A networks, that consists of seven adjacent hexagonal cells, with one UE served by each cell. UE positions and radio conditions are randomly generated, and the initial power allocation for each RB equals $\pi_{\min }(0.1 \mathrm{~W})$. System bandwidth equals $5 \mathrm{MHz}$. Thus, $25 \mathrm{RBs}$ are available in each cell. The maximum transmission power per cell $P_{\max }$ is 
set to $43 \mathrm{dBm}$ or $20 \mathrm{~W}$. At the first iteration, the dual variables $\lambda_{k, i, n}(0), \forall k \in$ $\mathcal{K}(i), \forall i \in \mathcal{I}, \forall n \in \mathcal{N}$, and $\nu_{i}(0), \forall i \in \mathcal{I}$, are assigned initial positive values. The evolution of $\widehat{\pi}_{i, 1}$ along with the number of iterations is shown in Fig. 1(a), where $\widehat{\pi}_{i, 1}$ is the logarithm of the transmission power allocated by the cell $i$ to the RB 1. In addition, the number of primal iterations and the number of dual iterations per round are shown in Fig. 1(b).

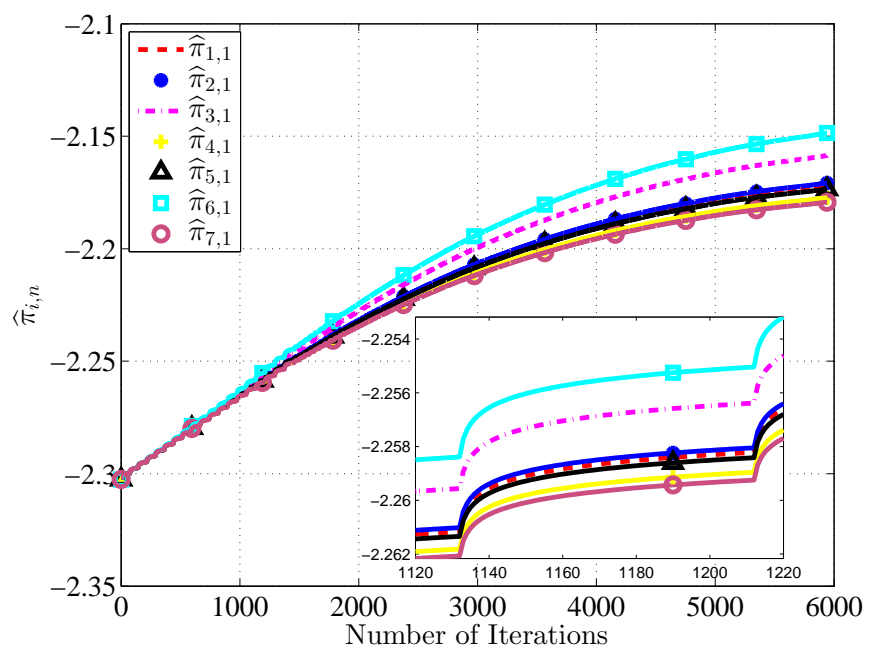

(a) Primal variables $\widehat{\pi}_{i, n}$

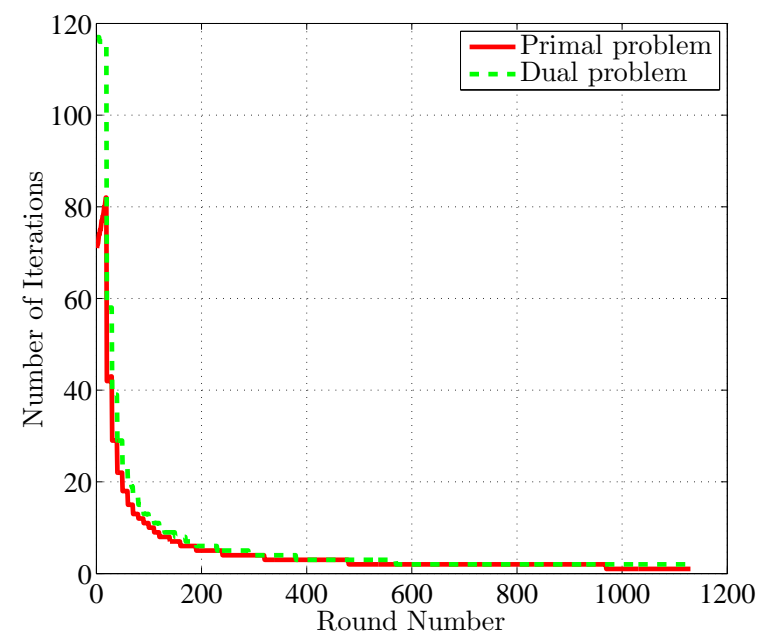

(b) Primal and dual iterations per round

Figure 1: Primal variables and number of iterations 
We notice that for the centralized power allocation approach, the primal problem requires approximately 6000 iterations to converge. As shown in Fig. 1(b), 1100 rounds are required to reach the optimal values of the primal and the dual variables. The zoomed box within Fig. 1(a) shows the evolution of $\widehat{\pi}_{i, n}$ versus the number of primal iterations for a given round t. The values of $\widehat{\pi}_{i, n}$ are calculated using the dual variables obtained at the round $(t-1)$. We also notice that the number of primal iterations and the number of dual iterations decreases with the number of rounds. When $t$ increases, the impact of Lagrange prices $\lambda_{k, i, n}(t)$ and $\nu_{i}(t)$ on the primal variables calculation is reduced, and the number of primal iterations required for the primal problem to converge becomes lower. The same behavior is noticed for the number of dual iterations when the number of rounds increases.

For the same simulated scenario, we also show the dual variables $\lambda_{k, i, n}$ and $\nu_{i}$ versus the number of dual iterations in Fig. 2(a) and Fig. 2(b), respectively. We notice that approximately 8000 iterations are required for the dual problem to converge. At a given round $t$, the Lagrange prices $\lambda_{k, i, n}$ and $\nu_{i}$ are updated using the most recent values of the primal variables. The zoomed boxes within Fig. 2(a) and Fig. 2(b) show the evolution of $\lambda_{k, i, n}$ and $\nu_{i}$ versus the number of iterations, respectively. These values are updated until $\Delta \lambda_{k, i, n}$ and $\Delta \nu_{i}$ become less than $\epsilon$. Convergence of the centralized power allocation problem occurs when two conditions are satisfied: first, the difference between the updated primal variables at round $t$ and their values at round $(t-1)$ is less than $\epsilon$.

370 Second, the difference between the updated primal variables at round $t$ and their values at round $(t-1)$ is less than $\epsilon$.

\subsection{Decentralized Power Allocation}

The same scenario in 7.1 is also simulated in this paragraph to evaluate the performance and convergence of the decentralized power allocation approach.

375 The evolution of the downlink transmission power allocated by all the cells to a given RB is shown in Fig. 3(a).

The initial value of the downlink transmission power allocated to each RB 


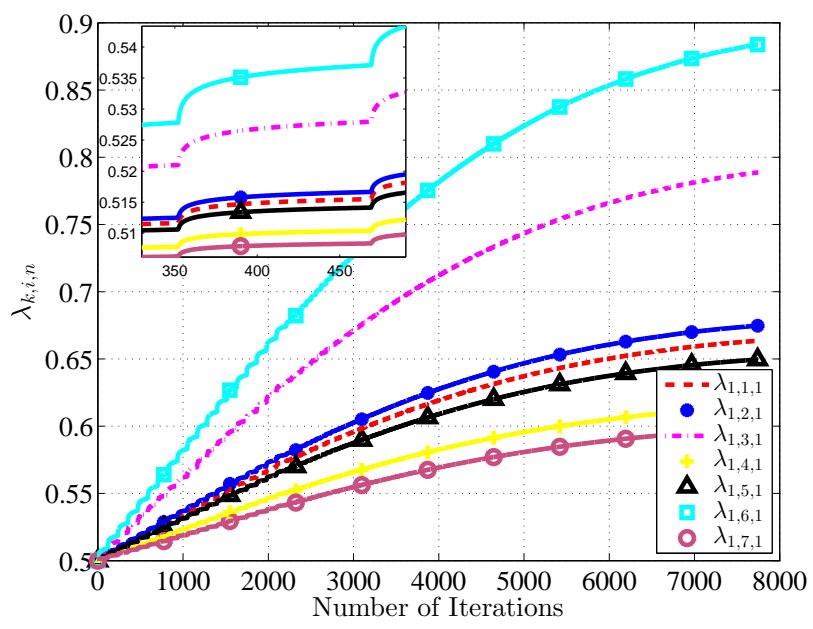

(a) Lagrange prices $\lambda_{k, i, n}$

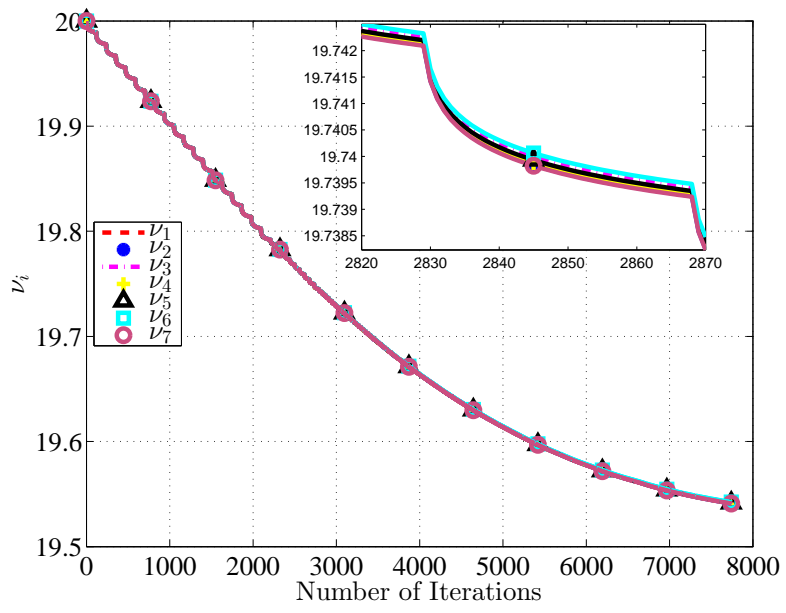

(b) Lagrange prices $\nu_{i}$

Figure 2: Convergence of the dual variables

equals $\pi_{\min }(0.1 \mathrm{~W})$. This allocation satisfies the constraints of the minimal downlink transmission power per RB and that of the maximum transmission power per cell. Each cell $i$ seeks maximizing its own utility function $U_{i}$ by adjusting the transmission power allocated to the available RBs. It also estimates the interference due to the usage of the same RBs by the neighboring cells. As shown in Fig. 3(a), each cell starts increasing the downlink transmission power 


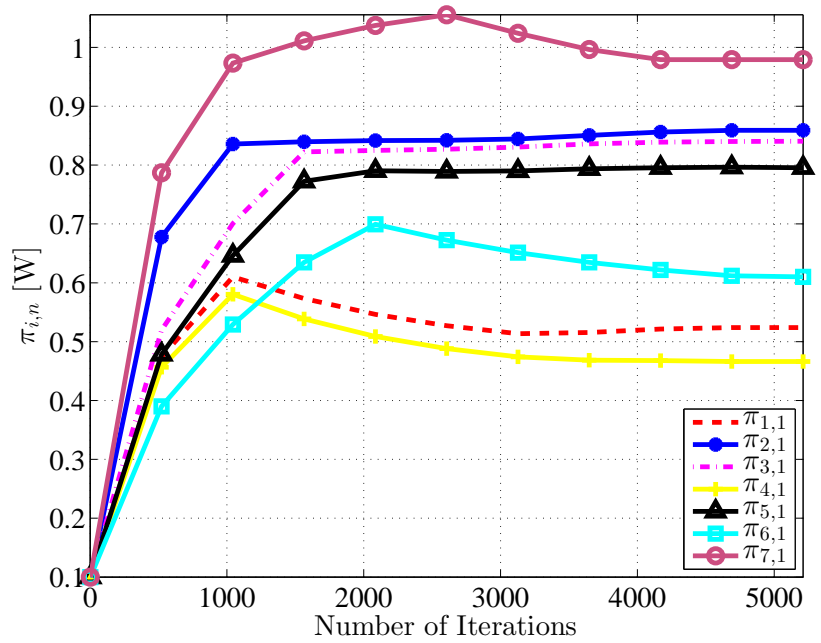

(a) $\pi_{i, n}$ versus the number of iterations

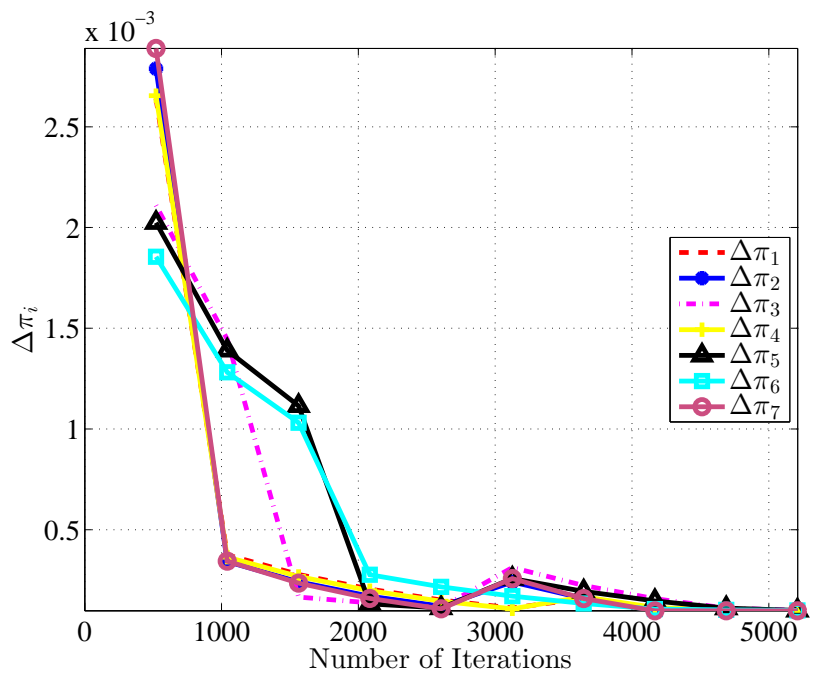

(b) $\Delta \pi_{i}$ versus the number of iterations

Figure 3: Convergence of the transmission power for the decentralized approach

allocated to its RBs, and then the transmission power converges after a given number of iterations. At convergence, the partial derivative of the objective function $U_{i}$ with respect to $\pi_{i, n}$ becomes negligible. The difference between the updated power allocation vector $\left(\pi_{i, 1}, \pi_{i, 2}, \ldots, \pi_{i, N}\right)$ at iteration $(t+1)$ and the power vector at iteration $\mathrm{t}$ becomes less than $\epsilon$. 
We also show the evolution of the power vector difference $\Delta \pi_{i}, \forall i \in \mathcal{I}$, defined show that $\Delta \pi_{i}, \forall i \in \mathcal{I}$, decreases when the number of iterations increases. The impact of the subgradient projection iterations on the downlink transmission power $\pi_{i, n}, \forall i \in \mathcal{I}, \forall n \in \mathcal{N}$, becomes smaller as more iterations are performed. Power convergence is achieved when $\Delta \pi_{i}, \forall i \in \mathcal{I}$, becomes less than $\epsilon$. In fact, downlink transmission power $\pi_{i, n}$ is modified becomes negligible.

\subsection{Comparison with State-of-the-Art Resource Allocation Approaches}

We also compare the performance of our proposed centralized and decentralized resource and power allocation approaches with that of state-of-the-art resource and power allocation approaches [36] such as the frequency reuse-1 model, the frequency reuse- 3 model, FFR, SFR, and a single cell resource and power allocation approach [12]. Note that our centralized approach searches for the optimal resource and power allocation. It is considered as a reference approach when comparing the performance of heuristic algorithms and distributed approaches.

The frequency reuse-1 model allows the usage of the same frequency spectrum simultaneously in all the network cells. Moreover, homogeneous power allocation is performed. In the frequency reuse-3 model, one third of the available spectrum is used in each cell in a cluster of three adjacent cells. Interference problems are eliminated, but the spectral efficiency is reduced. FFR and SFR techniques divide each cell into a cell-center and a cell-edge zones, and set restrictions on resource usage and power allocation in each zone. For all the compared techniques, resource allocation is performed according to Theorem 5.1.

\subsubsection{Spectral Efficiency}

415

We investigate the impact of the compared techniques on the spectral efficiency. Simulation results, including the $95 \%$ confidence interval, are shown in Fig. 4(a). 


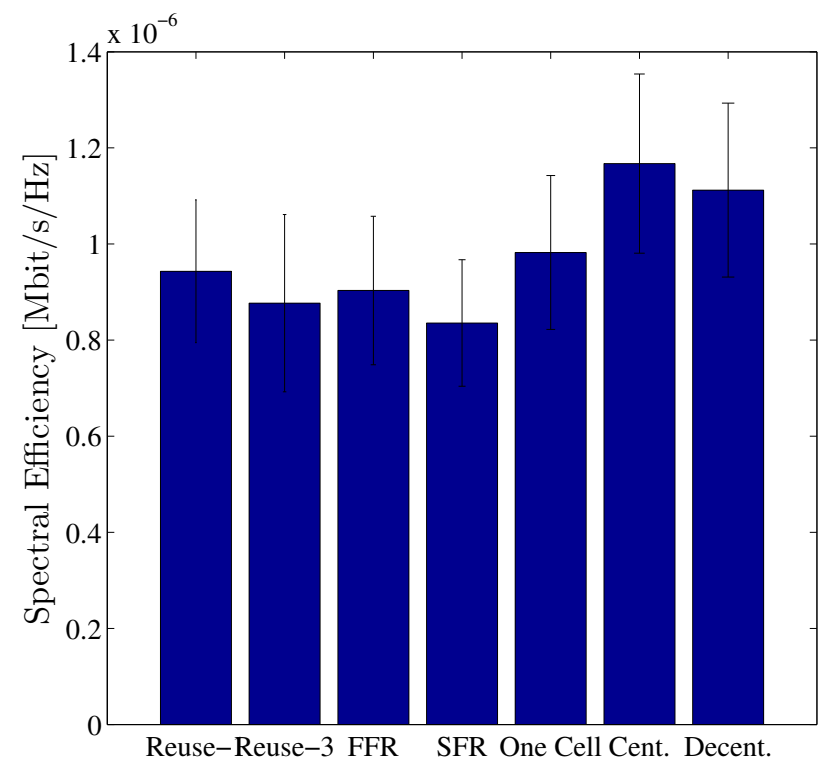

(a) Spectral efficiency

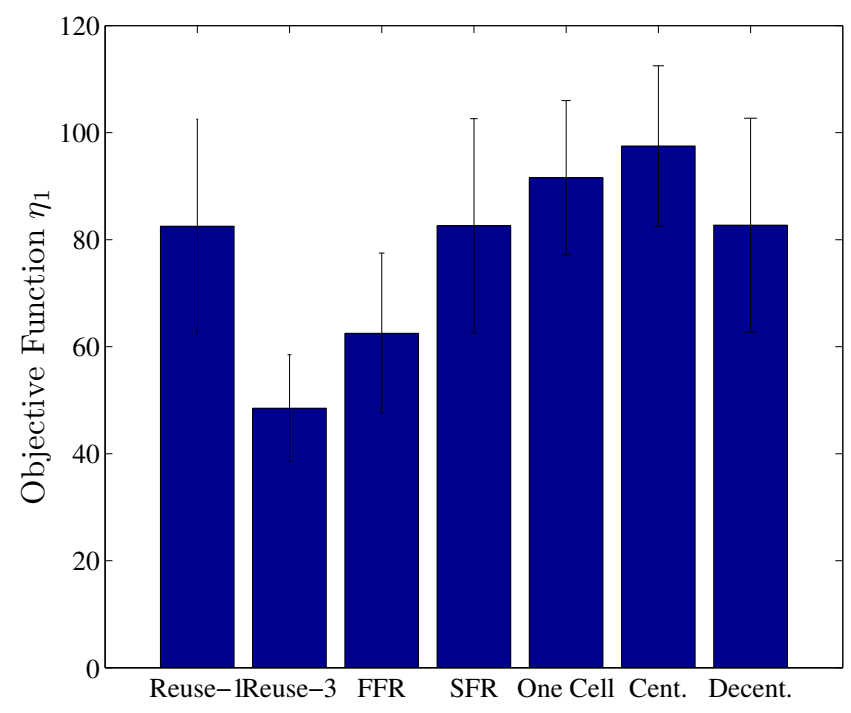

(b) Objective function $\eta_{1}$

Figure 4: Comparison with state-of-the-art approaches

Our proposed centralized resource allocation approach offers the highest spectral efficiency, since the optimal resource and power allocation is guaran- 
that of the centralized approach, due to the lack of information about resource usage in the neighboring cells. Nevertheless, the spectral efficiency for both the centralized and the decentralized approaches is greater than that of FFR, SFR, and the single-cell resource and power allocation approach [12] displayed as "One Cell" in Fig. 4. In fact, the static resource allocation between cell zones, and the quantified transmission power levels do not allow performing flexible resource allocation in a manner that satisfies UE needs in each cell. Concerning the single-cell approach, it does not take inter-cell interference problems into account; however, its spectral efficiency is slightly higher than that of reuse-1 model because it searches for the optimal resource and power allocation locally in each cell.

\subsubsection{Objective Function}

We also compare the objective function $\eta_{1}$ given in (6a) for the different resource and power allocation techniques. Simulation results are shown in 435 Fig. 4(b).

We notice that our centralized approach shows the highest objective function $\eta_{1}$. In fact, it finds the optimal power allocation for the entire system, while taking into account constraints related to resource usage and to the maximum downlink transmission power per cell. It outperforms the decentralized approach where each cell strives to maximize its own utility function independently of the other cells, and the single-cell approach where inter-cell interference problems are not taken into account when solving the resource and power allocation problem.

\subsection{Centralized Versus Decentralized Complexity Comparison}

We evaluate the computational complexity of our centralized and decentralized resource and power allocation approaches. For the centralized and decentralized approaches, resource allocation is performed according to Theorem 5.1, and it is equivalent to one operation. The complexity of each approach equals 
the number of required operations multiplied by the complexity of a single operation, denoted by $T_{o p}$. The complexity of the centralized approach is given by:

$$
O\left[\left(\mathrm{nb}_{\text {primal }} \times|\mathcal{N}| \times(1+k)+\operatorname{nb}_{\text {dual }} \times(1+k \times|\mathcal{N}|)\right) \times|\mathcal{I}| \times T_{o p}\right] .
$$

Similarly, the decentralized approach complexity is given as follows:

$$
O\left(\text { nb }_{\text {iterations }} \times|\mathcal{I}| \times|\mathcal{N}| \times T_{o p}\right)
$$

where $n b_{\text {primal }}$ is the number of primal iterations and $n b_{\text {dual }}$ is the number of dual iterations required for convergence of the centralized approach. $k$ is the number of UEs per cell, and $n b_{\text {iterations }}$ is the number of iterations required for convergence of the decentralized approach.

We notice that the decentralized approach complexity is independent of the number of UEs per cell, contrarily to the centralized approach. The complexity of both techniques depends of the number of cells in the system and the number of RBs available in each cell. Moreover, the computational complexity of these approaches are evaluated under the same simulation scenario as in 7.1. The median number of operations required for the centralized and decentralized approaches are given in Table 2.

Table 2: Median number of operations per approach

\begin{tabular}{c|c} 
Approach & Number of operations \\
\hline Centralized & $3.02 \cdot 10^{8}$ \\
\hline Decentralized & $8.84 \cdot 10^{5}$
\end{tabular}

According to the results reported in Table 2, the number of operations required for the centralized resource and power allocation approach largely exceeds that of the decentralized approach. In fact, the centralized approach maximizes the objective function for the entire network, contrarily to the decentralized approach where each cell maximizes its objective function independently of the other cells. Therefore, the centralized approach guarantees the optimal solution at the expense of a high computational complexity. 


\section{Conclusion}

Resource and power allocation problem is a challenging problem for nowa-

465

the joint resource and power allocation problem, and formulate it as nonlinear optimization problems. The objective consists in maximizing system throughput, spectral efficiency, or energy efficiency under constraints related to the minimum throughput per UE, QoS parameters, and the maximum transmis-

470 sion power. However, these techniques fall short from considering the impact of inter-cell interference. Indeed, each cell solves its own resource and power allocation problem without taking into account resource usage and power allocation in the neighboring cells.

In this article, we formulated the joint resource and power allocation problem for multiuser OFDMA networks as a centralized optimization problem, where the objective consists in maximizing system throughput while guaranteeing throughput fairness between UEs. The joint problem is then decomposed into two independent problems: a resource allocation problem and a power allocation problem. Contrarily to the majority of the state-of-the-art approaches, neighboring cells is taken into account when managing the resource and power allocation. Moreover, we introduced a decentralized power allocation approach based on game theory. The players are the cells, and each cell aims at maximizing its own utility function regardless of the decisions made by the other cells. Simulation results prove the convergence of our proposed approaches, and show the positive impact of our centralized and decentralized resource allocation approaches on system performance. In a future work, we will consider the formulation of a multi-cell and multi-objective resource and power allocation problem, where we address the compromise between spectral efficiency and 490 energy efficiency. 


\section{Appendix I}

\section{Upper Bound of the Objective Functions Difference}

Given Jensen's inequality and the concavity of the logarithmic function, we have:

$$
\begin{aligned}
& \log \left(\frac{\sum_{n \in \mathcal{N}} \theta_{k, n} \cdot \rho_{k, i, n}}{|\mathcal{N}|}\right) \geq \frac{\sum_{n \in \mathcal{N}} \log \left(\theta_{k, n} \cdot \rho_{k, i, n}\right)}{|\mathcal{N}|} \\
\Rightarrow & \log \left(\sum_{n \in \mathcal{N}} \theta_{k, n} \cdot \rho_{k, i, n}\right) \geq \frac{\sum_{n \in \mathcal{N}} \log \left(\theta_{k, n} \cdot \rho_{k, i, n}\right)}{|\mathcal{N}|}+\log (|\mathcal{N}|),
\end{aligned}
$$

the objective function $\eta$ can be written as:

$$
\begin{aligned}
\eta & =\sum_{i \in \mathcal{I}} \sum_{k \in \mathcal{K}(i)} \log \left(\sum_{n \in \mathcal{N}} \theta_{k, n} . \rho_{k, i, n}\right) \\
& \geq \frac{1}{|\mathcal{N}|} \sum_{i \in \mathcal{I}} \sum_{k \in \mathcal{K}(i)} \sum_{n \in \mathcal{N}} \log \left(\theta_{k, n} \cdot \rho_{k, i, n}\right)+|\mathcal{K}| \cdot \log (|\mathcal{N}|) .
\end{aligned}
$$

Since $\frac{1}{|\mathcal{N}|}$ and $|\mathcal{K}| \cdot \log (|\mathcal{N}|)$ are constant terms, maximizing the objective function of problem (4) is achieved by maximizing the following term:

$$
\begin{aligned}
& \sum_{i \in \mathcal{I}} \sum_{k \in \mathcal{K}(i)} \sum_{n \in \mathcal{N}} \log \left(\theta_{k, n} . \rho_{k, i, n}\right)= \\
& \sum_{i \in \mathcal{I}} \sum_{k \in \mathcal{K}(i)} \sum_{n \in \mathcal{N}}\left(\log \left(\theta_{k, n}\right)+\log \left(\rho_{k, i, n}\right)\right) .
\end{aligned}
$$

In order to decompose the joint problem into two independent problems, we evaluate the gap between the original objective function $\eta$ and the function given in (30). It is evident that there exists a gap between our objective function $\eta$ and the function (30) that we will maximize in the following sections. However, maximizing these two functions is equivalent as long as the gap between them is bounded. For this reason, we demonstrate the existence of a finite upper bound on the following difference:

$$
0 \leq \log \left(\sum_{n \in \mathcal{N}} \theta_{k, n} \cdot \rho_{k, i, n}\right)-\sum_{n \in \mathcal{N}} \log \left(\theta_{k, n} . \rho_{k, i, n}\right) \leq B
$$


Let $\phi_{n}=\theta_{k, n} \cdot \rho_{k, i, n}>0$, and suppose that $a \leq \phi_{n} \leq b$. Thus, there exists $0 \leq \gamma_{n} \leq 1$ such that $\phi_{n}=\gamma_{n} a+\left(1-\gamma_{n}\right) b$.

$$
\begin{aligned}
& \log \left(\sum_{n \in \mathcal{N}} \phi_{n}\right)-\sum_{n \in \mathcal{N}} \log \left(\phi_{n}\right) \\
& =\log \left(\sum_{n \in \mathcal{N}}\left(\gamma_{n} a+\left(1-\gamma_{n}\right) b\right)\right)-\sum_{n \in \mathcal{N}} \log \left(\gamma_{n} a+\left(1-\gamma_{n}\right) b\right) \\
& \leq \log \left(\sum_{n \in \mathcal{N}}\left(\gamma_{n} a+\left(1-\gamma_{n}\right) b\right)\right)-\sum_{n \in \mathcal{N}}\left(\gamma_{n} \log (a)+\left(1-\gamma_{n}\right) \log (b)\right) \\
& =\log \left(a\left(\sum_{n \in \mathcal{N}} \gamma_{n}\right)+b\left(|\mathcal{N}|-\sum_{n \in \mathcal{N}}\left(\gamma_{n}\right)\right)\right)-\log (a)\left(\sum_{n \in \mathcal{N}} \gamma_{n}\right) \\
& -\log (b)\left(|\mathcal{N}|-\sum_{n \in \mathcal{N}}\left(\gamma_{n}\right)\right) .
\end{aligned}
$$

Taking $p=\sum_{n \in \mathcal{N}} \gamma_{n}$ and $q=|\mathcal{N}|-\sum_{n \in \mathcal{N}} \gamma_{n}$, we have $0 \leq p \leq|\mathcal{N}|$, $0 \leq q \leq|\mathcal{N}|$, and $p+q=|\mathcal{N}|$. Consequently, we have:

$$
\begin{aligned}
& \log \left(\sum_{n \in \mathcal{N}} \phi_{n}\right)-\sum_{n \in \mathcal{N}} \log \left(\phi_{n}\right) \\
& \leq \log (a p+b q)-p \log (a)-q \log (b) \\
& \leq \max _{p}(\log (a p+b(|\mathcal{N}|-p))-p \log (a)-(|\mathcal{N}|-p) \log (b))=B .
\end{aligned}
$$

For fixed $a$ and $b$, let us denote:

$$
g(p)=\log (a p+b(|\mathcal{N}|-p))-p \log (a)-(|\mathcal{N}|-p) \log (b) .
$$

$g$ is defined, differentiable, and concave on $[0,|\mathcal{N}|]$. Its first derivative is given by:

$$
g^{\prime}(p)=\frac{a-b}{a p+b(|\mathcal{N}|-p)}-\log (a)+\log (b) .
$$


$g(p)$ attains its maximal value $B$ for a $p_{0}$ that satisfies: $g^{\prime}\left(p_{0}\right)=0$. Thus, we have $B=g\left(p_{0}\right)$, where $p_{0}$ is given by:

$$
p_{0}=\frac{1}{\log (a)-\log (b)}+\frac{b \cdot|\mathcal{N}|}{b-a} .
$$

Therefore, the considered difference is bounded by $B$, which is a finite bound.

\section{Appendix II}

\section{Proof of Theorem 5.1}

We consider the objective function (12a), which can be written as follows:

$$
\begin{aligned}
& \left(\eta_{2}\right)_{i}=\sum_{k \in \mathcal{K}(i)} \sum_{n \in \mathcal{N}} \log \left(\theta_{k, n}\right) \\
& =\log \left(\prod_{\substack{k \in \mathcal{K}(i) \\
n \in \mathcal{N}}} \theta_{k, n}\right) .
\end{aligned}
$$

Since the logarithmic function is monotonically increasing, the maximization of $\left(\eta_{2}\right)_{i}$ becomes equivalent to the maximization of the term $\prod_{\substack{k \in \mathcal{K}(i) \\ n \in \mathcal{N}}} \theta_{k, n}$. We consider the following cases:

1. Let us assume that:

$$
\sum_{k \in \mathcal{K}(i)} \theta_{k, n}<\sum_{n \in \mathcal{N}} \theta_{k, n}, \forall k \in \mathcal{K}(i), \forall n \in \mathcal{N}
$$

We suppose that $\theta_{k, n}, \forall k \in \mathcal{K}(i), \forall n \in \mathcal{N}$ is an optimal solution to the resource allocation problem (12) i.e., this solution maximizes the objective function (12a). For this solution, we assume that:

$$
\exists k \in \mathcal{K}(i) / \sum_{n \in \mathcal{N}} \theta_{k, n}<1
$$


We define $\epsilon>0$ as follows:

$$
\epsilon=1-\sum_{n \in \mathcal{N}} \theta_{k, n}
$$

and we demonstrate that this solution is not an optimal solution to problem (12) using the proof by contradiction. In fact, we define a set of $\theta_{k, n}^{\prime}$ variables as given in the following:

$$
\theta_{k, n}^{\prime}= \begin{cases}\theta_{k, n}, & \forall n \in \mathcal{N}, n \neq n_{1}, \forall k \in \mathcal{K}(i) \\ \theta_{k, n}+\epsilon, & \text { if } n=n_{1}, \forall k \in \mathcal{K}(i) .\end{cases}
$$

Therefore, we have:

$$
\prod_{\substack{k \in \mathcal{K}(i) \\ n \in \mathcal{N}}} \theta_{k, n}^{\prime}=\prod_{\substack{k \in \mathcal{K}(i) \\ n \in \mathcal{N}}} \theta_{k, n}+\epsilon \cdot \prod_{\substack{k \in \mathcal{K}(i) \\ n \in \mathcal{N}}} \theta_{k, n}>\prod_{\substack{k \in \mathcal{K}(i) \\ n \in \mathcal{N}}} \theta_{k, n}
$$

and the assumption made in (34) is false, since it does not maximize the objective function (12a). Consequently, we have:

$$
\begin{aligned}
\sum_{n \in \mathcal{N}} \theta_{k, n} & =1, \forall k \in \mathcal{K}(i) \\
\Rightarrow \sum_{k \in \mathcal{K}(i)} \sum_{n \in \mathcal{N}} \theta_{k, n} & =|\mathcal{K}(i)| .
\end{aligned}
$$

Since the sum of all the $\theta_{k, n}$ variables is constant, the term $\prod_{\substack{k \in \mathcal{K}(i) \\ n \in \mathcal{N}}} \theta_{k, n}$ reaches its maximum when all the variables $\theta_{k, n}$ are equal i.e.,

$$
\theta_{k, n}=\frac{|\mathcal{K}(i)|}{|\mathcal{K}(i)| \cdot|\mathcal{N}|}=\frac{1}{|\mathcal{N}|}, \forall k \in \mathcal{K}(i), \forall n \in \mathcal{N}
$$

which is an optimal solution to the resource allocation problem (12). Ac- 
cording to (33):

$$
\begin{aligned}
& \sum_{k \in \mathcal{K}(i)} \theta_{k, n}<\sum_{n \in \mathcal{N}} \theta_{k, n}, \forall k \in \mathcal{K}(i), \forall n \in \mathcal{N} \\
\Rightarrow & \frac{|\mathcal{K}(i)|}{|\mathcal{N}|}<1 \\
\Rightarrow & |\mathcal{K}(i)|<|\mathcal{N}| .
\end{aligned}
$$

2. Similarly when:

$$
\sum_{n \in \mathcal{N}} \theta_{k, n}<\sum_{k \in \mathcal{K}(i)} \theta_{k, n}, \forall k \in \mathcal{K}(i), \forall n \in \mathcal{N}
$$

In this case, the optimal solution is given by:

$$
\theta_{k, n}=\frac{|\mathcal{N}|}{|\mathcal{K}(i)| \cdot|\mathcal{N}|}=\frac{1}{|\mathcal{K}(i)|}, \forall k \in \mathcal{K}(i), \forall n \in \mathcal{N}
$$

\section{Appendix III}

\section{Proof of Proposition 6.2}

To prove the super-modularity of the present game, we need to verify the conditions in 6.2. First, the strategy space $S_{i}$ is obviously a compact convex set of $\mathbb{R}^{N}$. Hence, it suffices to verify the super-modularity of the utility function as there are no constraint policies for $\mathcal{G}$ :

$$
\begin{gathered}
\frac{\partial U_{i, n}}{\partial \pi_{l, n} \partial \pi_{i, n}}= \\
\frac{1}{\log \left(1+\sigma_{k, i, n}\right)} \frac{G_{k, i, n} G_{k, l, n}}{\left(N_{0}+\sum_{i^{\prime} \neq i} \pi_{i^{\prime}, n} G_{k, i^{\prime}, n}\right)^{2}}\left(\frac{\sigma_{k, i, n}}{\log \left(1+\sigma_{k, i, n}\right)}-1\right) .
\end{gathered}
$$

As $\frac{x}{\log (1+x)}>1$ for $x>0, \frac{\partial U_{i, n}}{\partial \pi_{l, n} \partial \pi_{i, n}} \geq 0, \forall l \in \mathcal{I}-\{i\}$ and $\forall n \in \mathcal{N}$. 


\section{References}

[1] 3GPP, Physical Layer Aspects for Evolved Universal Terrestrial Radio Access (UTRA) (Release 7), Tech. rep., 3GPP TR 25.814 V7.1.0 (2006).

[2] 3GPP, Evolved Universal Terrestrial Radio Access (E-UTRA): Physical Layer Procedures, Technical specification, 3GPP TS 36.213 V11.11.0 (December 2013).

[3] N. Hassan, M. Assaad, Optimal Fractional Frequency Reuse (FFR) and Resource Allocation in Multiuser OFDMA System, in: Int. Conf. Information and Communication Technologies, Karachi, 2009, pp. 88-92. doi:10.1109/ICICT.2009.5267207.

[4] Huawei, Soft Frequency Reuse Scheme for UTRANLTE (R1-050507), Technical report, 3GPP RAN WG1 no. 41, Athens, Greece (May 2005).

[5] M. Rahman, H. Yanikomeroglu, Multicell Downlink OFDM Subchannel Allocations Using Dynamic Intercell Coordination, in: IEEE Global Telecommunications Conf., 2007, pp. 5220-5225. doi:10.1109/GLOCOM. 2007.990.

[6] M. Rahman, H. Yanikomeroglu, Enhancing Cell-Edge Performance: A Downlink Dynamic Interference Avoidance Scheme with Inter-Cell Coordination, IEEE Trans. Wireless Commun. 9 (4) (2010) 1414-1425. doi: 10.1109/TWC. 2010.04 .090256 .

[7] M. Yassin, S. Lahoud, M. Ibrahim, K. Khawam, A Downlink Power Control Heuristic Algorithm for LTE Networks, in: $21^{\text {st }}$ Int. Conf. Telecommunications, Lisbon, 2014, pp. 323-327. doi:10.1109/ICT.2014.6845132.

${ }_{530}$ [8] M. Yassin, S. Lahoud, M. Ibrahim, K. Khawam, D. Mezher, B. Cousin, Non-Cooperative Inter-Cell Interference Coordination Technique for Increasing Through Fairness in LTE Networks, in: IEEE $81^{\text {st }}$ Vehicular Technology Conf., Glasgow, 2015. 
[9] M. Chiang, C. W. Tan, D. Palomar, D. O'Neill, D. Julian, Power Control By Geometric Programming, IEEE Trans. Wireless Commun. 6 (7) (2007) 2640-2651. doi:10.1109/TWC. 2007.05960.

[10] J. Tang, D. So, E. Alsusa, K. Hamdi, Resource Efficiency: A New Paradigm on Energy Efficiency and Spectral Efficiency Tradeoff, IEEE Trans. Wireless Commun. 13 (8) (2014) 4656-4669.

[11] R. Loodaricheh, S. Mallick, V. Bhargava, Energy-Efficient Resource Allocation for OFDMA Cellular Networks with User Cooperation and QoS Provisioning, IEEE Trans. Wireless Commun. 13 (11) (2014) 6132-6146.

[12] C. Xiong, G. Li, S. Zhang, Y. Chen, S. Xu, Energy and Spectral-Efficiency Tradeoff in Downlink OFDMA Networks, IEEE Trans. Wireless Commun. 10 (11) (2011) 3874-3886. doi:10.1109/TWC.2011.091411.110249.

[13] T. Quek, Z. Lei, S. Sun, Adaptive Interference Coordination in MultiCell OFDMA Systems, in: IEEE $20^{\text {th }}$ Int. Symp. Personal, Indoor and Mobile Radio Commun., 2009, pp. 2380-2384. doi:10.1109/PIMRC. 2009. 5450369 .

[14] Y. Umeda, K. Higuchi, Efficient Adaptive Frequency Partitioning in OFDMA Downlink with Fractional Frequency Reuse, in: Int. Symp. Intelligent Signal Processing and Commun. Systems, 2011, pp. 1-5. doi: 10.1109/ISPACS. 2011.6146189.

[15] L. Venturino, A. Zappone, C. Risi, S. Buzzi, Energy-Efficient Scheduling and Power Allocation in Downlink OFDMA Networks with Base Station Coordination, IEEE Trans. Wireless Commun. 14 (1) (2015) 1-14. doi: 10.1109/TWC.2014.2323971.

[16] C. Xiong, G. Li, S. Zhang, Y. Chen, S. Xu, Energy-Efficient Resource Allocation in OFDMA Networks, IEEE Trans. Commun. 60 (12) (2012) 
[17] J. Yu, G. Li, C. Yin, S. Tang, X. Zhu, Multi-Cell Coordinated Scheduling and Power Allocation in Downlink LTE-A Systems, in: IEEE $80^{\text {th }}$ Vehicular Technology Conf., Vancouver, 2014, pp. 1-5. doi:10.1109/VTCFall. 2014.6966061.

[18] M. Aboul Hassan, E. Sourour, S. Shaaban, Novel Resource Allocation Algorithm for Improving Reuse One Scheme Performance in LTE Networks, in: $21^{\text {st }}$ Int. Conf. Telecommunications, Lisbon, 2014, pp. 166-170. doi:10.1109/ICT.2014.6845101.

[19] D. W. K. Ng, R. Schober, Resource Allocation and Scheduling in MultiCell OFDMA Systems with Decode-and-Forward Relaying, IEEE Trans. Wireless Commun. 10 (7) (2011) 2246-2258.

[20] C. Bae, W. Stark, End-to-End Energy-Bandwidth Tradeoff in Multihop Wireless Networks, IEEE Trans. Information Theory 55 (9) (2009) 40514066. doi:10.1109/TIT.2009. 2025556.

[21] D. T. Ngo, S. Khakurel, T. LeNgoc, Joint Subchannel Assignment and Power Allocation for OFDMA Femtocell Networks, IEEE Trans. Wireless Commun. 13 (1) (2014) 342-355.

[22] J. Zheng, Y. Cai, Y. Liu, Y. Xu, B. Duan, X. Shen, Optimal Power Allocation and User Scheduling in Multicell Networks: Base Station Cooperation Using a Game-Theoretic Approach, IEEE Trans. Wireless Commun. 13 (12) (2014) 6928-6942.

[23] S. Sadr, R. Adve, Partially-Distributed Resource Allocation in Small-Cell Networks, IEEE Trans. Wireless Commun. 13 (12) (2014) 6851-6862.

[24] J. S. Wang, J. H. Lee, J. C. Park, I. Song, Y. H. Kim, Combining of Cyclically Delayed Signals: A Low-Complexity Scheme for PAPR Reduction in OFDM Systems, IEEE Trans. Broadcasting 56 (4) (2010) 577-583. doi:10.1109/TBC. 2010.2060174. 
[25] E. Dahlman, S. Parkvall, J. Skold, 4G LTE and LTE-Advanced for Mobile Broadband, 1st Edition, Elsevier, Oxford, 2011.

[26] A. Zappone, E. Jorswieck, Energy Efficiency in Wireless Networks via Fractional Programming Theory, Foundations and Trends in Commun. and Information Theory 11 (4) (2015) 185-396.

[27] F. Kelly, Charging and Rate Control for Elastic Traffic, European Trans. Telecommunications 8 (1) (1997) 33-37.

[28] S. Boyd, L. Vandenberghe, Convex Optimization, 7th Edition, Vol. 7, UK: Cambridge University Press, Cambridge, 2009.

[29] D. Palomar, M. Chiang, A Tutorial on Decomposition Methods for Network Utility Maximization, IEEE J. Selected Areas in Commun. 24 (8) (2006) 1439-1451. doi:10.1109/JSAC.2006.879350.

[30] M. Chiang, Geometric Programming for Communication Systems, Foundations and Trends of Commun. and Information Theory 2 (1-2) (2005) 1 $-156$.

[31] J. B. Rosen, Existence and Uniqueness of Equilibrium Points for Concave n-Person Games, Econometrica J. 33 (1965) 520-534.

[32] D. Topkis, Supermodularity and Complementarity, Princeton University Press, Princeton, 1998.

[33] Topkis, Equilibrium Points in Non-Zero Sum n-Person Sub-modular Games, SIAM J. Control Optim. 17 (6) (1979) 773-787.

[34] D. Yao, S-Modular Games, with Queueing Applications, Queueing Systems 610 $21(3-4)(1995)$ 449-475.

[35] D. P. Palomar, Convex Primal Decomposition for Multicarrier Linear MIMO Transceivers, IEEE Trans. Signal Processing 53 (12) (2005) 46614674. 
[36] M. Yassin, M. AboulHassan, S. Lahoud, M. Ibrahim, D. Mezher, B. Cousin, E. Sourour, Survey of ICIC Techniques in LTE Networks under Various Mobile Environment Parameters, Accepted for publication in Springer Wireless Networks. 